\title{
Ecological assessment of wetland ecosystems of northern Kazakhstan on the basis of hydrochemistry and algal biodiversity
}

\author{
Sophia S. Barinova ${ }^{1 *}$, Eibi Nevo $^{1}$, TATIAna M. Bragina $^{2}$ \\ ${ }^{1}$ Institute of Evolution, University of Haifa, Mount Carmel, Haifa 31905, Israel \\ ${ }^{2}$ Russian University of Kazakhstan, Kustanaiskaya Oblast, Naurzum Rayon, \\ Karamendy, Altynsarin Str., 45-1, Kazakhstan
}

\begin{abstract}
We studied diversity of algae and cyanobacteria in the wetlands of protected natural lakes with salinity ranging from 0.19 up to 32.7 in the arid/semiarid regions of Northern Kazakhstan. In plankton and periphyton of 34 lakes, we found 254 species belonging to 113 genera of 8 algal divisions. The diversity in arid regions is represented by widespread species of diatoms, green algae, and cyanobacteria in similar proportions. Alkaliphiles, among the indicators of acidification, and betamesosaprobionts, among the indicators of saprobity, predominated. The indices of saprobity in lakes varied from 1.47 to 2.7, reflecting low-trophic and low anthropogenically disturbed wetlands. Oligohalobes-indifferents are most common. Highly diverse algal communities were found irrespective of various levels of mineralization. As a consequence of aridization, salinity increase suppressed algal diversity. The mineralization was the most important variable defining the diversity levels, irrespective of the type and location of wetland lakes in the arid regions.
\end{abstract}

Keywords: Algae, cyanobacteria, aridization, diversity, salinity, wetland, water quality, Kazakhstan

\section{Introduction}

In arid regions, aquatic environments experience a stressful impact of high concentrations of mineral and organic substances due to high evaporation rates (SUBYANI 2005). Algal habitats are characterized by a high amplitude salinity variation that in large lakes suppresses algal diversity (HAMMER 1986). For example lower bacterial diversity (RUSZNYÁK et al. 2008) and algal species richness (Ács et al. 2003) were found in the open water area of Lake Velencei (where the conductivity is about $2.5-3.5 \mathrm{mS} \mathrm{cm}^{-1}$ averagely) than in the bog-like area of the lake (where the conductivity is about $1.5-2.3 \mathrm{mS} \mathrm{cm}^{-1}$ on average). Many algal species are indicators of environmental conditions reflecting the influence of

* Corresponding author, e-mail: barinova@research.haifa.ac.il

Copyright $^{\circledR} 2011$ by Acta Botanica Croatica, the Faculty of Science, University of Zagreb. All rights reserved. 
salinity on aquatic communities and the regional flora as a whole. A decrease of algal diversity is in turn related to reduced productivity of aquatic ecosystem and thereby of the trophic level of wetlands. It is well known that an increase of salinity to 20, suppress the diversity of lake biota (HAMMER 1986).

The arid regions of Central Asia and Middle East occupy a considerable part of Eurasia (KÖPPEN and GEIGER 1953). In Kazakhstan, the arid and semiarid dry grasslands to deserts are widespread in the upper reaches of the Ob' River Basin and the Turkestan Desert (BRAGINA and BRAgin, 2002). The hydrographic network of this territory is developed slightly, closed and has no constant drainage (SKLIARENKO, 2006). During spring high water, channels are filled with water that reaches lakes and spreads wide over the steppe. The freshwater and the salt waterlakes have depths not exceeding 2.5-3 $\mathrm{m}$ and have the typical features of all reservoirs of arid territories, a cyclic hydrological mode where the periods of filling and drying repeat each 12-15 years. Large highly mineralized lakes Balkhash, Tengiz, Issyk Kul' and Karakul', as well as the Aralian and Caspian seas, are confined to this climatic area (HAMMER 1986).

Phytogeographically, this region is situated near the boundary of the Irano-Turanian province and the province north of it (TAKHTAJAN 1978). A large number of lakes in this area are protected on account of their importance for biodiversity conservation (BRAGINA and BRAGIN 2002).

We previously studied algal biodiversity in respect to ecological assessment of the wetlands (BARINOVA et al. 2002) and compared it with other arid regions (BARINOVA et al. 2009). Here we continue studying the diversity of algal communities and its relation to salinity.

\section{Material and methods}

This research was based upon 98 samples of phytoplankton and periphyton collected in the North Kazakhstan Region in October 1999 and May-June 2000. Altogether 34 lakes were sampled, as well as the mouth of Karasu River near Lake Tuntugur, Jailmo Well near Lake Kulykol, and Jarsor Brook near Lake Jarsor located in the northern Kazakhstan arid area (Fig. 1). The water level in the studied shallow lakes depends on the climate and on snow melt, and is related to the complete or partial drying of some lakes in dry years. The studied shallow lakes have a permanent depth of 0.5-2.0 m. They become overgrown by underwater and surface vegetation, and exhibit high hydrogen sulphide content in their water, which varies in seasons and in lake gulfs (BRAGINA and BRAGIN 2002). Water salinity in the lakes varies from fresh to salty, with sulfates and chlorides prevailing.

The samples were taken by scooping up for phytoplankton and by scratching for periphyton and were fixed in 3\% formaldehyde (WHITTON et al. 1991). Algae were studied with Swift and Amplival dissecting microscopes under magnifications of 740-1850 and were photographed with the digital camera Inspector 1 . The diatoms were prepared with the peroxide technique (SWIFT 1967) modified for glass slides (BARINOVA 1988, 1997).

In parallel with sampling for algae we measured electrical conductivity, total dissolved solids, and pH with HANNA HI 9813, and DPC2, concentration of $\mathrm{N}_{-} \mathrm{NO}_{3}$ with $\mathrm{HANNA}$ HI 93728 and phosphates with HACH spectrophotometer, as well as salinity by the argentometric method (APHA 1998); pH measurements were made down to 0.15. 


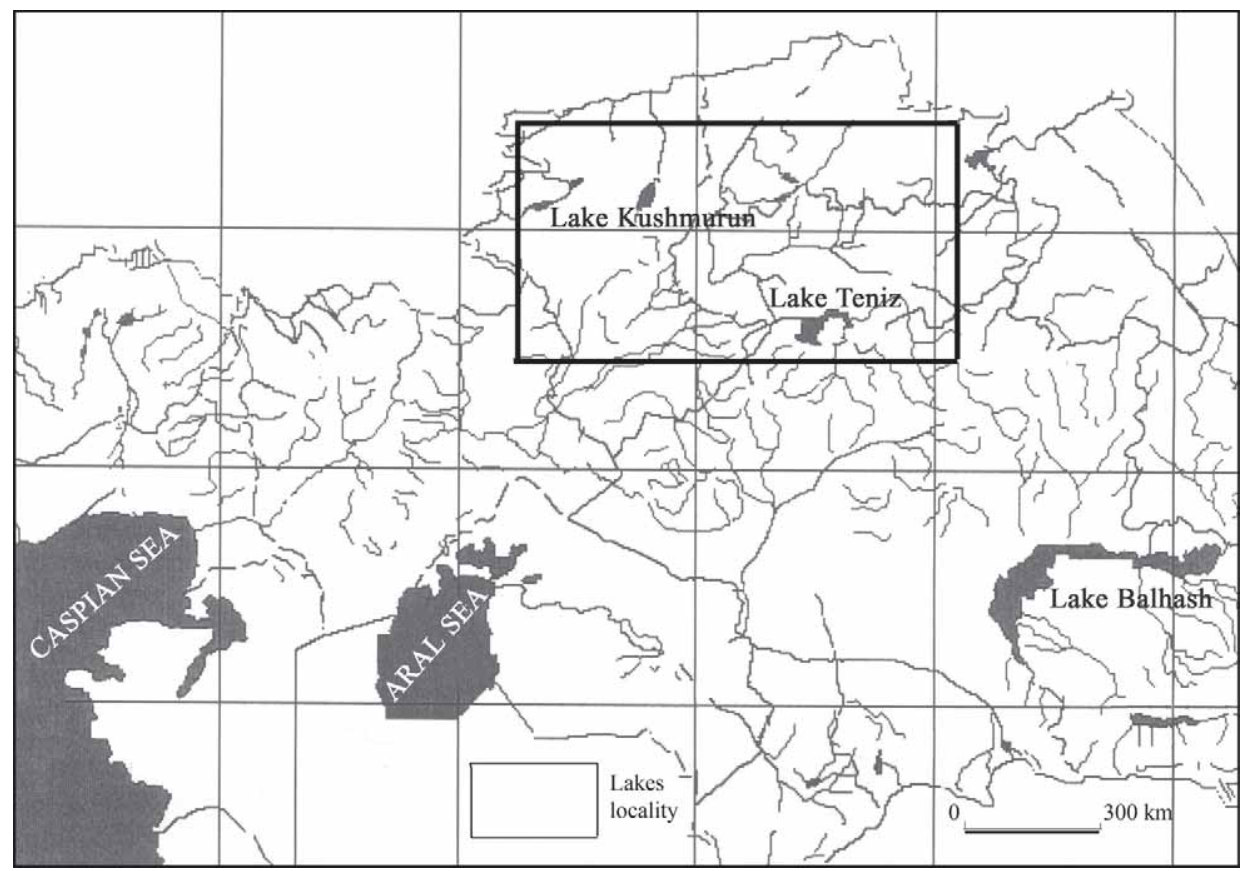

Fig. 1. Lakes and wetland region in the Northern Kazakhstan.

The assessment of $\beta+\gamma$ radioactive pollution of water samples was performed with detector-indicator of radioactivity QUARTEX RD 8901.

The algal abundances were assessed on the basis of a 6-score scale (KoRDE 1956, BARINOVA et al. 2006).

The taxonomy follows the systems adopted in the »Süswasserflora von Mitteleuropa « (EtTl 1978; Starmach 1985; EtTl and Gartner 1988; Krammer and Lange-Bertalot 1991a, b, c, d; KomÁreK and ANAGnostidis 1998) and MATTOX and STEWART (1984) system for green algae, with additions for individual taxa in Gollerbach et al. (1953), Kisselev (1954), Popova (1966), Vinogradova et al. (1980), Palamar-Mordvintseva (1982), Krammer (1985, 2000), Moshkova and Gollerbach (1986), Lange-Bertalot and Krammer (1987), MefFert (1987), Komárek and Anagnostidis (1989), Popovsky and Pfiester (1990), Barber and Carter (1996), Hegewald (2000), Rumrich et al. (2000).

The ecological and geographic characteristics of algae are obtained from the database compiled by the author for freshwater algae as a basis for statistical analysis of algal biodiversity distribution over ecological gradients (BARINOVA 2000, BARINOVA et al. 2000, 2006).

Our ecological analysis has revealed a grouping of freshwater algae in respect to $\mathrm{pH}$, salinity and saprobity as well as the other habitat conditions. Each group was separately assessed with respect to its significance for bioindications. Species that respond predictably to environmental variables can be used as bioindicators reflecting the response of aquatic ecosystems to eutrophication, $\mathrm{pH}$ levels (acidifications), salinity and organic pollutants. 
Distribution of species sensitive to $\mathrm{pH}$ and suitable as bioindicators for acidity is analyzed in accordance with the classification of HUSTEDT (1938-1939). This classification system is divided into $\mathrm{pH}$-related groups, from alkalibiontes to acidobiontes.

The bioindication of salinity is based on the classification system by HUSTEDT (1957) with groups ranging from polyhalobes to oligohalobes-halophobes according to KOLBE's (1927) system of halobity.

There are several alternative approaches to assessment of saprobity (adaptation to excessive levels of nutrients), with that of PANTLE and BUCK (1955) modified by SLÁDEČEK $(1973,1986)$ being found most suitable for the present analysis. The indicators of saprobity are assigned to four groups according to their saprobity index values (S) ranging from polysaprobes $(S=3.5-4.0)$ to xenosaprobes $(S=0-0.5)$. The indices of saprobity are obtained as a function of saprobic species numbers and their relative abundances:

$$
\mathrm{S}=\Sigma \mathrm{sh} / \Sigma \mathrm{h} \quad(\text { Eq. } 1)
$$

where $\mathrm{S}$ indicates index of saprobity for algal community; $\mathrm{s}$ - species-specific saprobity level; $\mathrm{h}$ - abundance on the 6-scores scale (after KoRDE 1956).

For phytogeographic analysis, species ranges were plotted and assigned to phytogeographic divisions of TAKHTAJAN (1978).

Statistical methods were used in comparative floristic approaches (NovAKOVSKY 2004) for clarifying of algal flora similarity in the natural protected wetlands in the semi-arid climate of the Northern Kazakhstan. The percent disagreement was calculated by Ward's method in Statistica 6.0 Program.

Ecological classification of water quality was based on a combination of hydrochemical variables and the indices of saprobity (ROMANENKO et al. 1990, BARINOVA et al. 2006). The status of water objects was assessed as sum of all data integrated in the functional model of an aquatic ecosystem (BARINOvA et al. 2006). Gamma and beta radiation were studied as background variables.

\section{Results}

\section{Influence of ecological conditions on biodiversity of algae in the arid region wetlands of Kazakhstan}

Because of the remoteness of Kazakhstan's protected areas, algal diversity there has remained virtually unstudied. The lakes that we studied can be considered as typical for this region. Their salinity (as the saltiness or dissolved salt content of a body of water) varies from 0.19 to $32.7 \mathrm{NaCl} \mathrm{g} \mathrm{L}^{-1}$ (Tab. 1) increasing during the summer dry period and contains not only chlorides but also sulphates as regional norm. The acidity varies from slightly acid to alkaline, whereas the concentration of nitrates and phosphates attests to a sufficient trophic base for algal development (Tab. 1). At the same time, the saprobity indices of PANTLE and BuCK (1955) modified by SLÁDEČEK (1973) varied from 1.65 in the Gr. Karakamys Lake and 1.47 in the Karasu River mouth to 2.7 in the lakes Tahtakul and Aksuat, which indicates a lack of appreciable anthropogenic impact. 
Tab. 1. Environmental conditions, species number and index of saprobity in the lakes of the Kazakhstan arid regions in 1999-2000; classes of salinity and groups of salinity indicators according to HUSTEDT (1957): mh, mesohalobes; hl, oligohalobes - halophilous; i, oligohalobes - indifferent; saprobity index S according to SLÁDEČEK (1973), and equation (1).

\begin{tabular}{|c|c|c|c|c|c|c|c|c|c|c|c|}
\hline $\begin{array}{l}\text { No of } \\
\text { Lake }\end{array}$ & Lake & $\begin{array}{l}\text { Conduc- } \\
\text { tivity, } \\
\mathrm{mSm} / \mathrm{cm}\end{array}$ & $\begin{array}{l}\mathrm{NaCl} \\
\mathrm{g} \mathrm{L}^{-1}\end{array}$ & $\begin{array}{l}\text { Class of } \\
\text { Salinity }\end{array}$ & $\mathrm{pH}$ & $\begin{array}{c}\mathrm{P}-\mathrm{PO}_{4}{ }^{3-} \\
\mathrm{mg} \mathrm{L}^{-1}\end{array}$ & $\begin{array}{c}\mathrm{N}_{-\mathrm{NO}_{3}}^{-}, \\
\mathrm{mg} \mathrm{L}^{-1}\end{array}$ & $\begin{array}{c}\text { No. of } \\
\text { algal } \\
\text { species }\end{array}$ & $\begin{array}{c}\text { Saprobity } \\
\text { Index S }\end{array}$ & $\begin{array}{l}\text { Group of } \\
\text { salinity } \\
\text { indicators }\end{array}$ & $\begin{array}{c}\text { Group of } \\
\text { similarity } \\
\text { on the tree }\end{array}$ \\
\hline 1 & Aike & 11.73 & 6.79 & III & 7.01 & 0.16 & 3.4 & 19 & 1.98 & $\mathrm{hl}$ & 1 \\
\hline 2 & Aksuat & $1.16-6.95$ & $0.69-3.94$ & IV & $6.16-6.56$ & $0.01-0.02$ & $1.0-1.4$ & 1 & 2.70 & - & 2 \\
\hline 3 & Alpash & 8.72 & 4.92 & IV & 7.05 & 0.01 & 1.0 & 13 & 1.74 & $\mathrm{i}$ & 2 \\
\hline 4 & Annovskoe & $0.7-0.79$ & $0.43-0.45$ & IV & $6.87-7.11$ & 0.01 & $1.2-2.0$ & 22 & $1.71-1.81$ & hl & 2 \\
\hline 5 & Balykty & 4.33 & 2.43 & IV & 7.84 & 0.02 & 1.1 & 26 & 2.21 & $\mathrm{i}$ & 1 \\
\hline 6 & Batpakkol & 0.37 & 0.21 & IV & 7.29 & 0.26 & 2.3 & 2 & 2.0 & hl & 2 \\
\hline 7 & Biesoygan & 0.851 & 0.52 & IV & 6.76 & 0.39 & 2.1 & 7 & 2.55 & $\mathrm{i}$ & 2 \\
\hline 8 & Bozshakol & $0.62-0.91$ & $0.38-0.55$ & IV & $6.43-7.37$ & $0.20-2.25$ & $1.7-8.5$ & 63 & $1.95-2.13$ & $\mathrm{hl}$ & 3 \\
\hline 9 & Chushkaly & 16.4 & 9.35 & III & 6.94 & 0.28 & 1.7 & 18 & 1.97 & $\mathrm{hl}+\mathrm{mh}$ & 1 \\
\hline 10 & Gr. Kak & 57.3 & 32.7 & II & 6.36 & 0.05 & 2.2 & 6 & 2.11 & hl & 2 \\
\hline 11 & Gr. Karakamys & $0.81-2.88$ & $0.46-1.7$ & IV & $6.59-6.66$ & $0.01-0.68$ & $0.8-1.2$ & 28 & $1.65-1.8$ & $\mathrm{i}+\mathrm{hl}$ & 2 \\
\hline 12 & Gr. Sankebay & 14.29 & 8.17 & III & 7.40 & 0.09 & 1.6 & 3 & 2.49 & $\mathrm{hl}$ & 2 \\
\hline 13 & Jaltyr & 11.47 & 6.52 & III & 6.77 & 1.0 & 1.0 & 6 & 1.86 & $\mathrm{i}$ & 2 \\
\hline 14 & Jaman & $0.37-0.41$ & $0.22-0.23$ & IV & $6.54-7.53$ & $0.03-0.08$ & $1.2-1.6$ & 57 & $1.78-2.10$ & $\mathrm{i}+\mathrm{hl}$ & 3 \\
\hline 15 & Jarken & 0.86 & 0.49 & IV & 6.74 & 0.03 & 0.9 & 22 & 2.00 & $\mathrm{i}+\mathrm{hl}$ & 2 \\
\hline 16 & Jarkol & $5.45-5.67$ & $3.1-3.42$ & IV & $7.26-7.38$ & $0.17-1.08$ & $2.3-4.1$ & 8 & $1.7-1.96$ & $\mathrm{i}$ & 2 \\
\hline 17 & Jilandy & 1.48 & 0.85 & IV & 7.15 & 0.68 & 1.2 & 4 & 2.05 & $\mathrm{i}+\mathrm{hl}$ & 2 \\
\hline 18 & Kamyshovoe & $0.37-0.43$ & $0.21-0.26$ & IV & $6.29-7.14$ & $0.02-0.11$ & $1.2-1.9$ & 79 & $1.86-1.9$ & $\mathrm{i}+\mathrm{hl}$ & 3 \\
\hline
\end{tabular}


Tab. 1. - continued

\begin{tabular}{|c|c|c|c|c|c|c|c|c|c|c|c|}
\hline $\begin{array}{l}\text { No of } \\
\text { Lake }\end{array}$ & Lake & $\begin{array}{l}\text { Conduc- } \\
\text { tivity, } \\
\mathrm{mSm} / \mathrm{cm}\end{array}$ & $\begin{array}{l}\mathrm{NaCl} \\
\mathrm{g} \mathrm{L}^{-1}\end{array}$ & $\begin{array}{l}\text { Class of } \\
\text { Salinity }\end{array}$ & $\mathrm{pH}$ & $\begin{array}{c}\mathrm{P}-\mathrm{PO}_{4}{ }^{3-} \\
\mathrm{mg} \mathrm{L}^{-1}\end{array}$ & $\begin{array}{c}\mathrm{N}^{-\mathrm{NO}_{3}^{-}}, \\
\mathrm{mg} \mathrm{L}^{-1}\end{array}$ & $\begin{array}{l}\text { No. of } \\
\text { algal } \\
\text { species }\end{array}$ & $\begin{array}{c}\text { Saprobity } \\
\text { Index S }\end{array}$ & $\begin{array}{l}\text { Group of } \\
\text { salinity } \\
\text { indicators }\end{array}$ & $\begin{array}{l}\text { Group of } \\
\text { similarity } \\
\text { on the tree }\end{array}$ \\
\hline 19 & $\begin{array}{l}\text { Karasu River, } \\
\text { near Lake } \\
\text { Tuntugur }\end{array}$ & 0.398 & 0.24 & IV & 6.86 & 0.23 & 1.4 & 2 & 1.47 & $\mathrm{hl}$ & 2 \\
\hline 20 & Koybagor & $1.1-1.16$ & $0.66-0.69$ & IV & $6.83-7.35$ & $0.07-0.27$ & $1.3-2.0$ & 112 & $1.95-2.04$ & $\mathrm{i}+\mathrm{hl}$ & 3 \\
\hline 23 & $\begin{array}{l}\text { Kulykol, } \\
\text { Well Jailma }\end{array}$ & 1.01 & 0.58 & IV & 7.28 & 0.06 & 1.0 & 3 & 2.35 & $\mathrm{i}+\mathrm{hl}$ & 2 \\
\hline 24 & Kushmurun & 30.1 & 18.0 & III & 6.77 & 0.1 & 3.5 & 8 & 2.08 & $\mathrm{i}+\mathrm{hl}$ & 2 \\
\hline 25 & Majbalyk & $1.95-3.23$ & $1.11-1.78$ & IV & $6.92-7.4$ & $0.0-0.02$ & $1.4-2.2$ & 4 & 2.04 & $\mathrm{i}$ & 2 \\
\hline 26 & Sankebay & 14.68 & 8.8 & III & 8.15 & 0.54 & 5.4 & 5 & 1.93 & $\mathrm{i}$ & 2 \\
\hline 30 & Sultan & 3.04 & 1.73 & IV & 6.73 & 0.0 & 0.9 & 5 & 2.15 & $\mathrm{i}$ & 2 \\
\hline 31 & Suly & 0.328 & 0.19 & IV & 7.13 & 1.77 & 1.4 & 7 & 1.84 & $\mathrm{i}$ & 2 \\
\hline 32 & Tahtakul & $0.99-2.3$ & $0.56-1.38$ & IV & $6.35-6.79$ & $0.03-0.48$ & $1.1-1.8$ & 7 & $1.8-2.7$ & $\mathrm{i}$ & 2 \\
\hline 33 & Teniz & $2.52-6.99$ & $1.43-3.98$ & IV & $6.66-6.69$ & $0.0-0.04$ & $1.0-1.5$ & 61 & $1.87-1.96$ & $\mathrm{i}+\mathrm{hl}$ & 3 \\
\hline 34 & Tyuntugur & $1.03-1.16$ & $0.63-0.7$ & IV & $6.66-7.53$ & $0.01-0.26$ & $1.5-2.0$ & 58 & $1.88-2.12$ & $\mathrm{i}+\mathrm{hl}$ & 3 \\
\hline
\end{tabular}


Our taxonomic analysis revealed 254 species from eight taxonomical divisions, among which diatoms slightly prevail over green and blue-green species (Tab. 2, Fig. 2a). For 13 species we indicate only generic assignments because certain critical features, such as sexual forms in Spirogyra, are lacking in our material.

Tab. 2. Ecology and geographical distribution of algae and cyanobacteria in Kazakhstan lakes. Ecological types: Habitat: B - benthic; P - planktonic; $\mathrm{P}-\mathrm{B}$ - planktonic-benthic; $\mathrm{T}$ - temperature; temp - temperate; eterm - eurytermic; warm - warm-water; cool - cool-water; Reo - reophility and oxygenation; st - standing water; str - stream; D - saprobity categories of WATANABE (1986); es - eurysaprob; sx - saproxen; sp - saprophil; S - saprobity categories of SLÁDEČEK (1986); o oligosaprob; o- $\alpha$ - oligo-alfa-mesosaprob; o- $\beta$ - oligo-beta-mesosaprob; $\beta$ - beta-mesosaprob; $\beta$ - $\alpha$ - beta-alfa-mesosaprob; $\alpha$-alfa-mesosaprob; $\alpha-\beta$-alfa-beta-mesosaprob; $x$-xenosaprob; $\alpha-\rho$ - alfa-meso-polysaprob; $\rho$ - polysaprob; Hal - halobity; $\mathrm{mh}$ - mesohalobe; i - oligohalobious-indifferent; $\mathrm{hl}$ - oligohalobious-halophilous; $\mathrm{hb}$ - oligohalobious-halophobous; $\mathrm{ph}$ - polyhalobe; $\mathrm{pH}$ - acidophility; ind - indifferent; alf - alkaliphil; acf - acidophil; alb - alkalibiont; Geo - chorological types; $\mathrm{mt}$ - Mediterranean; $\mathrm{k}$ - cosmopolite; $\mathrm{b}$ - Boreal; Pt - Paleotropical; $\mathrm{Nt}$ - Neotropical; Ha - Holarctic. »-«no data. Numbers of lakes as in Table 1.

\begin{tabular}{|c|c|c|c|c|c|c|c|c|c|c|}
\hline No & Taxon & No of Lake & Habitat & $\mathrm{T}$ & Reo & $\mathrm{D}$ & S & Hal & $\mathrm{pH}$ & Geo \\
\hline \multicolumn{11}{|c|}{ Cyanoprokaryota } \\
\hline 1 & $\begin{array}{l}\text { Anabaena constricta (Szaf.) } \\
\text { Geitl. }\end{array}$ & 21 & $\mathrm{P}-\mathrm{B}$ & - & - & - & $\mathrm{p}$ & - & - & $\mathrm{Ha}$ \\
\hline 2 & Anabaena contorta Bachm. & 1 & $\mathrm{P}$ & - & st-str & - & - & - & - & $\mathrm{k}$ \\
\hline 3 & $\begin{array}{l}\text { Anabaena flos-aquae (Lyngb.) } \\
\text { Bréb. f. flos-aquae }\end{array}$ & 18 & $\mathrm{P}$ & - & st & - & $\beta$ & $\mathrm{i}$ & - & $\mathrm{k}$ \\
\hline 4 & $\begin{array}{l}\text { Anabaena flos-aquae f. } \\
\text { jacutica (Nyg.) Elenk. }\end{array}$ & 8 & $\mathrm{P}$ & - & - & - & - & $\mathrm{i}$ & - & $\mathrm{b}$ \\
\hline 5 & Anabaena minima Tschernov & 33 & - & - & - & - & - & - & - & $\mathrm{b}$ \\
\hline 6 & Anabaena sp. & $18,28,33$ & - & - & - & - & - & - & - & - \\
\hline 7 & Anabaena spiroides Kleb. & 34 & $\mathrm{P}$ & - & st-str & - & o & $\mathrm{i}$ & - & $\mathrm{k}$ \\
\hline 8 & Anabaena variabilis Kütz. & 9 & P-B & - & st & - & - & $\mathrm{mh}$ & - & $\mathrm{k}$ \\
\hline 9 & $\begin{array}{l}\text { Aphanizomenon flos-aquae } \\
\text { (L.) Ralfs }\end{array}$ & $6,8,20$ & $\mathrm{P}$ & - & - & - & $\beta$ & hl & - & $\mathrm{k}$ \\
\hline 10 & $\begin{array}{l}\text { Aphanothece clathrata W. et } \\
\text { G. S. West }\end{array}$ & $\begin{array}{l}1,4,8,14,20 \\
24,27\end{array}$ & $\mathrm{P}$ & - & - & - & $\beta$ & hl & - & $\mathrm{k}$ \\
\hline 11 & $\begin{array}{l}\text { Aphanothece stagnina } \\
\text { (Spreng.) A. Br. }\end{array}$ & $8,20,21$ & P-B & - & - & - & o & hl & ind & $\mathrm{k}$ \\
\hline 12 & $\begin{array}{l}\text { Chroococcus limneticus } \\
\text { Lemm. }\end{array}$ & 20 & $\mathrm{P}$ & - & - & - & o & - & - & $\mathrm{k}$ \\
\hline 13 & $\begin{array}{l}\text { Chroococcus minor (Kütz.) } \\
\text { Näg. }\end{array}$ & 33 & B & - & - & - & $o-\beta$ & - & - & $\mathrm{k}$ \\
\hline 14 & $\begin{array}{l}\text { Chroococcus minutus (Kütz.) } \\
\text { Näg. }\end{array}$ & 20 & $\mathrm{P}$ & - & - & - & - & - & - & $\mathrm{k}$ \\
\hline 15 & $\begin{array}{l}\text { Chroococcus turgidus (Kütz.) } \\
\text { Näg. }\end{array}$ & $\begin{array}{l}1,3,5,8,21 \\
33,35\end{array}$ & P-B & - & - & - & o & hl & alf & $\mathrm{k}$ \\
\hline 16 & Chroococcus vacuolatus Skuja & 33 & P-B & - & - & - & - & - & - & $\begin{array}{l}\mathrm{b}, \\
\mathrm{mt}\end{array}$ \\
\hline
\end{tabular}


Tab. 2. - continued

\begin{tabular}{|c|c|c|c|c|c|c|c|c|c|c|}
\hline No & Taxon & No of Lake & Habitat & $\mathrm{T}$ & Reo & $\mathrm{D}$ & S & Hal & $\mathrm{pH}$ & Geo \\
\hline 17 & Chroococcus varius A. Br. & 4,8 & B & - & - & - & o- $\beta$ & - & - & $\mathrm{k}$ \\
\hline 18 & $\begin{array}{l}\text { Coelomoron pusillum } \\
\text { (Van Goor) Komárek }\end{array}$ & $\begin{array}{l}1,4,8,14,21 \\
33\end{array}$ & $\mathrm{P}$ & temp & st & - & $\alpha$ & - & - & $\mathrm{k}$ \\
\hline 19 & $\begin{array}{l}\text { Coelosphaerium } \\
\quad \text { kuetzingianum Näg. }\end{array}$ & 15 & $\mathrm{P}$ & - & - & - & - & $\mathrm{i}$ & - & $\mathrm{k}$ \\
\hline 20 & $\begin{array}{l}\text { Coelosphaerium minutissimum } \\
\text { Lemm. }\end{array}$ & 21 & $\mathrm{P}$ & - & - & - & - & hl & - & $\mathrm{k}$ \\
\hline 21 & Gloeotrichia pisum (Ag.) Thur. & 4,18 & B & - & - & - & - & hl & ind & $\mathrm{k}$ \\
\hline 22 & Gomphosphaeria aponina Kütz. & 28,33 & $\mathrm{P}$ & - & - & - & - & hl & alf & $\mathrm{k}$ \\
\hline 23 & $\begin{array}{l}\text { Lyngbya aestuarii (Mert.) } \\
\text { Leibm. }\end{array}$ & 28 & P-B & - & - & - & - & - & - & $\mathrm{k}$ \\
\hline 24 & Lyngbya contorta Lemm. & 1 & - & - & - & - & - & hl & - & $\mathrm{Ha}$ \\
\hline 25 & Lyngbya sp. & 15,18 & - & - & - & - & - & - & - & - \\
\hline 26 & Merismopedia minima Beck & $\begin{array}{l}18,20,28,33 \\
34\end{array}$ & B & - & - & - & - & - & - & $\mathrm{Ha}$ \\
\hline 27 & Merismopedia smithii De Toni & $14,18,20$ & $\mathrm{P}$ & cool & - & - & - & - & - & $\mathrm{Ha}$ \\
\hline 28 & $\begin{array}{l}\text { Merismopedia tenuissima } \\
\text { Lemm. }\end{array}$ & $\begin{array}{l}1,8,9,14,20 \\
33\end{array}$ & P-B & - & - & - & $\beta$ & hl & - & $\mathrm{k}$ \\
\hline 29 & $\begin{array}{l}\text { Microcystis aeruginosa (Kütz.) } \\
\text { Kütz. }\end{array}$ & 7,8 & $\mathrm{P}$ & - & - & - & $\beta$ & hl & - & $\mathrm{k}$ \\
\hline 30 & $\begin{array}{l}\text { Microcystis pulverea f. } \\
\text { delicatissima (W. et G. S. } \\
\text { West) Elenk. }\end{array}$ & 1 & $\mathrm{P}$ & - & - & - & - & $\mathrm{i}$ & - & $\mathrm{k}$ \\
\hline 31 & Nostoc kihlmanii Lemm. & 30 & $\mathrm{P}$ & cool & st & - & - & $\mathrm{i}$ & ind & $\mathrm{Ha}$ \\
\hline 32 & $\begin{array}{l}\text { Oscillatoria brevis Kütz. ex } \\
\text { Gom. }\end{array}$ & 10 & P-B & - & st & - & $\alpha$ & - & - & $\mathrm{k}$ \\
\hline 33 & Oscillatoria limosa (Roth) Ag. & 11 & P-B & - & st-str & - & $\alpha$ & hl & - & $\mathrm{k}$ \\
\hline 34 & $\begin{array}{l}\text { Oscillatoria princeps Vauch. } \\
\text { ex Gom. }\end{array}$ & 10,22 & P-B & - & st-str & - & $\alpha$ & - & - & $\mathrm{k}$ \\
\hline 35 & Phormidium ambiguum Gom. & 11 & B & - & st-str & - & - & $\mathrm{i}$ & ind & $\mathrm{k}$ \\
\hline 36 & $\begin{array}{l}\text { Phormidium autumnale (Ag.) } \\
\text { Gom. }\end{array}$ & $20,30,33$ & B & - & st-str & - & $\beta$ & - & - & $\mathrm{k}$ \\
\hline 37 & $\begin{array}{l}\text { Phormidium paulsenianum } \\
\text { B.-Peters. }\end{array}$ & 28 & B & - & - & - & - & $\mathrm{ph}$ & - & $\mathrm{Ha}$ \\
\hline 38 & Phormidium retzii Ag. ex Gom. & 10 & B & - & st-str & - & o & - & - & $\mathrm{k}$ \\
\hline 39 & $\begin{array}{l}\text { Rhabdogloea scenedesmoides } \\
\text { (Nyg.) Komárek et Anagn. }\end{array}$ & 8 & $\mathrm{P}$ & cool & st & - & - & - & - & $\mathrm{Ha}$ \\
\hline 40 & Spirulina major Kütz. ex Gom. & 10 & $\mathrm{P}$ & - & st & - & - & - & - & $\mathrm{k}$ \\
\hline 41 & Synechocystis sallensis Skuja & 20 & P-B & cool & st & - & o & - & & $\begin{array}{l}\mathrm{Ha}, \\
\mathrm{Nt}\end{array}$ \\
\hline 42 & Tolypothrix sp. & 21 & - & - & - & - & - & - & - & - \\
\hline 43 & $\begin{array}{l}\text { Woronichinia compacta } \\
\text { (Lemm.) Komárek et Hindák }\end{array}$ & $1,18,21,27$ & $\mathrm{P}$ & cool & st & - & - & - & & $\begin{array}{c}\mathrm{Ha}, \\
\mathrm{Pt}\end{array}$ \\
\hline
\end{tabular}


Tab. 2. - continued

\begin{tabular}{|c|c|c|c|c|c|c|c|c|c|c|}
\hline No & Taxon & No of Lake & Habitat & $\mathrm{T}$ & Reo & $\mathrm{D}$ & S & Hal & $\mathrm{pH}$ & Geo \\
\hline \multicolumn{11}{|c|}{ Bacillariophyta } \\
\hline 44 & Achnanthes brevipes Ag. & $11,13,28$ & B & - & - & - & - & hl & alf & $\mathrm{k}$ \\
\hline 45 & $\begin{array}{l}\text { Achnanthes gibberula var. } \\
\text { interrupta Poretzky et } \\
\text { Anisimova }\end{array}$ & 20 & B & - & - & - & - & hl & - & $\mathrm{k}$ \\
\hline 46 & $\begin{array}{l}\text { Achnanthes lanceolata (Bréb. in } \\
\text { Kütz.) Grun. in Cl. et Grun. }\end{array}$ & $5,8,22,29,34$ & P-B & warm & st-str & sx & o & $\mathrm{i}$ & alf & $\mathrm{k}$ \\
\hline 47 & Achnanthes minutissima Kütz. & $\begin{array}{l}3,11,18,20 \\
27-29,33\end{array}$ & B & eterm & st-str & es & o & $\mathrm{i}$ & alf & $\mathrm{k}$ \\
\hline 48 & $\begin{array}{l}\text { Amphipleura pellucida (Kütz.) } \\
\text { Kütz. }\end{array}$ & 5,33 & B & - & st & - & $0-\alpha$ & $\mathrm{i}$ & alf & $\mathrm{k}$ \\
\hline 49 & $\begin{array}{l}\text { Amphora coffeaeformis (Ag.) } \\
\text { Kütz. }\end{array}$ & $3,22,33$ & B & - & st-str & - & - & $\mathrm{mh}$ & - & $\mathrm{k}$ \\
\hline 50 & $\begin{array}{l}\text { Amphora commutata Grun. in } \\
\text { V. H. }\end{array}$ & 3 & B & - & - & - & - & hl & - & $\mathrm{k}$ \\
\hline 51 & $\begin{array}{l}\text { Amphora holsatica Hust. in } \\
\text { Pasch. }\end{array}$ & 11 & $\mathrm{P}$ & - & st-str & - & - & hl & - & $\mathrm{k}$ \\
\hline 52 & Amphora ovalis (Kütz.) Kütz. & $\begin{array}{l}3,8,11,14,18 \\
20,33,34\end{array}$ & B & temp & st-str & sx & $\alpha-\beta$ & $\mathrm{i}$ & alf & $\mathrm{k}$ \\
\hline 53 & $\begin{array}{l}\text { Amphora pediculus (Kütz.) } \\
\text { Grun. ex A. Schmidt }\end{array}$ & $\begin{array}{l}5,8,16,20,21 \\
33,34\end{array}$ & B & temp & - & es & $\alpha-\beta$ & $\mathrm{i}$ & alf & $\mathrm{k}$ \\
\hline 154 & Amphora veneta Kütz. & $4,8,10,14,28$ & B & - & - & es & $\alpha-p$ & $\mathrm{i}$ & alf & $\mathrm{k}$ \\
\hline 55 & Asterionella formosa Hass. & $4,8,18$ & $P$ & - & - & sx & & $\mathrm{i}$ & alf & $\mathrm{k}$ \\
\hline 56 & $\begin{array}{l}\text { Aulacoseira granulata (Ehrb.) } \\
\text { Sim. }\end{array}$ & $8,33,34$ & P-B & cool & st-str & es & $\beta$ & $\mathrm{i}$ & alf & $\mathrm{k}$ \\
\hline 57 & Aulacoseira italica (Ehrb.) Sim. & 34 & P-B & cool & st-str & es & $\beta$ & $\mathrm{i}$ & alf & $\mathrm{k}$ \\
\hline 58 & $\begin{array}{l}\text { Caloneis amphisbaena (Bory) } \\
\text { Cl. }\end{array}$ & $\begin{array}{l}5,8,12,20 \\
28-30,34\end{array}$ & B & - & - & - & - & hl & alf & $\mathrm{k}$ \\
\hline 59 & Caloneis silicula (Ehrb.) $\mathrm{Cl}$. & $\begin{array}{l}5,8,11,18,20, \\
32\end{array}$ & $\mathrm{~B}$ & - & st & $\mathrm{sp}$ & $\beta$ & $\mathrm{i}$ & alf & $\mathrm{k}$ \\
\hline 60 & $\begin{array}{l}\text { Caloneis westii (W. Sm.) } \\
\text { Hendey }\end{array}$ & 5,33 & B & - & - & - & - & $\mathrm{mh}$ & - & $\mathrm{k}$ \\
\hline 61 & Campylodiscus noricus Ehrb. & 26,33 & B & - & - & - & o & $\mathrm{i}$ & alf & $\mathrm{k}$ \\
\hline 62 & Chaetoceros sp. & 9,33 & $\mathrm{P}$ & - & - & - & - & - & - & - \\
\hline 63 & Cocconeis placentula Ehrb. & $\begin{array}{l}9,11,15 \\
18-20,33\end{array}$ & P-B & temp & st-str & es & o & $\mathrm{i}$ & alf & $\mathrm{k}$ \\
\hline 64 & $\begin{array}{l}\text { Craticula cuspidata (Kütz.) } \\
\text { D. G. Mann }\end{array}$ & $5,20,33$ & B & temp & st & - & - & $\mathrm{i}$ & alf & $\mathrm{k}$ \\
\hline 65 & $\begin{array}{l}\text { Cyclostephanos dubius } \\
\text { (Fricke in A. Schmidt) Round }\end{array}$ & 20 & - & - & - & - & $\beta-\alpha$ & hl & alf & $\mathrm{k}$ \\
\hline 66 & $\begin{array}{l}\text { Cymatopleura librile (Ehrb.) } \\
\text { Pant. }\end{array}$ & $\begin{array}{l}5,7,89,14,18, \\
20,25,27,28, \\
34\end{array}$ & P-B & - & - & - & - & - & - & $\mathrm{k}$ \\
\hline
\end{tabular}


Tab. 2. - continued

\begin{tabular}{|c|c|c|c|c|c|c|c|c|c|c|}
\hline No & Taxon & No of Lake & Habitat & $\mathrm{T}$ & Reo & $\mathrm{D}$ & $\mathrm{S}$ & Hal & $\mathrm{pH}$ & Geo \\
\hline 67 & $\begin{array}{l}\text { Cymbella cornuta (Ehrb.) } \\
\text { R. Ross }\end{array}$ & $\begin{array}{l}8,14,18,27 \\
31,34\end{array}$ & B & - & - & - & - & $\mathrm{i}$ & alf & $\mathrm{k}$ \\
\hline 68 & Cymbella neocistula Krammer & 14 & B & - & st-str & sx & $\beta$ & $\mathrm{i}$ & alf & $\mathrm{k}$ \\
\hline 69 & Cymbella tumida (Bréb.) V. H. & $8,18,20$ & B & temp & - & sx & o & $\mathrm{i}$ & alf & $\mathrm{k}$ \\
\hline 70 & Cymbella turgidula Grun. & $\begin{array}{l}5,14,18,20 \\
33\end{array}$ & B & - & st-str & es & - & - & ind & $\mathrm{k}$ \\
\hline 71 & $\begin{array}{l}\text { Diatoma vulgare Bory var. } \\
\text { vulgare }\end{array}$ & $\begin{array}{l}18,20,22,27 \\
33,34\end{array}$ & P-B & - & st-str & - & $\mathrm{x}$ & $\mathrm{i}$ & ind & $\mathrm{k}$ \\
\hline 72 & $\begin{array}{l}\text { Diatoma vulgare var. } \\
\text { ehrenbergii (Kütz.) Grun. }\end{array}$ & 34 & B & - & - & - & - & $\mathrm{i}$ & alf & $\mathrm{k}$ \\
\hline 73 & Diploneis elliptica (Kütz.) Cl. & 23 & B & eterm & - & sx & o & $\mathrm{i}$ & alf & $\mathrm{k}$ \\
\hline 74 & Diploneis ovalis (Hilse) $\mathrm{Cl}$. & 24 & B & - & - & $\mathrm{sp}$ & & $\mathrm{i}$ & alb & $\mathrm{b}$ \\
\hline 75 & $\begin{array}{l}\text { Encyonema silesiacum } \\
\quad \text { (Bleisch. in Rabenh.) } \\
\text { D. G. Mann in Round et al. }\end{array}$ & $8,18,27,34$ & B & - & st-str & sx & o- $\beta$ & $\mathrm{i}$ & ind & $\mathrm{k}$ \\
\hline 76 & $\begin{array}{l}\text { Epithemia adnata (Kütz.) } \\
\text { Bréb. }\end{array}$ & $\begin{array}{l}5,8,14,18,20 \\
31,34\end{array}$ & B & temp & - & sx & $\beta$ & $\mathrm{i}$ & alf & $\mathrm{k}$ \\
\hline 77 & Epithemia sorex Kütz. & $14,18,20,33$ & B & temp & st & sx & $\beta$ & $\mathrm{i}$ & alf & $\mathrm{k}$ \\
\hline 78 & $\begin{array}{l}\text { Epithemia turgida (Ehrb.) } \\
\text { Kütz. }\end{array}$ & $\begin{array}{l}4,8,11,14,15 \\
18,20,29,31 \\
34\end{array}$ & B & temp & - & - & - & $\mathrm{i}$ & alf & $\mathrm{k}$ \\
\hline 79 & $\begin{array}{l}\text { Eunotia exigua (Bréb. ex } \\
\text { Kütz.) Rabenh. }\end{array}$ & 11 & B & - & - & es & o & $\mathrm{hb}$ & acf & $\mathrm{k}$ \\
\hline 80 & $\begin{array}{l}\text { Eunotia minor (Kütz.) Grun. } \\
\text { in V. H. }\end{array}$ & 18 & - & - & - & es & $\beta-\alpha$ & - & ind & $\mathrm{k}$ \\
\hline 81 & Eunotia monodon Ehrb. & 15 & B & - & - & & o & $\mathrm{hb}$ & acf & $\mathrm{k}$ \\
\hline 82 & $\begin{array}{l}\text { Eunotia pectinalis (O. Müll.) } \\
\quad \text { Rabenh. }\end{array}$ & 18,27 & B & - & - & sx & - & $\mathrm{hb}$ & acf & $\mathrm{k}$ \\
\hline 83 & $\begin{array}{l}\text { Eunotia praerupta Ehrb. var. } \\
\quad \text { praerupta }\end{array}$ & 18 & B & cool & st-str & sx & - & $\mathrm{hb}$ & acf & $\mathrm{k}$ \\
\hline 84 & $\begin{array}{l}\text { Eunotia praerupta var. bidens } \\
\text { (Ehrb.) Grun. in Cl. et Grun. }\end{array}$ & 18 & B & cool & - & - & - & $\mathrm{hb}$ & acf & $\mathrm{k}$ \\
\hline 85 & $\begin{array}{l}\text { Eunotia sibirica } \mathrm{Cl} \text {. in } \mathrm{Cl} \text {. et } \\
\text { Grun. }\end{array}$ & 11,31 & B & - & - & - & - & $\mathrm{i}$ & - & $\mathrm{b}$ \\
\hline 86 & $\begin{array}{l}\text { Fallacia pygmaea (Kütz.) } \\
\text { Stikle et Mann }\end{array}$ & 5,24 & B & - & - & es & $\alpha$ & $\mathrm{mh}$ & alf & $\mathrm{k}$ \\
\hline 87 & Fragilaria crotonensis Kitton & 3,8 & $\mathrm{P}$ & - & st & es & - & hl & alf & $\mathrm{k}$ \\
\hline 88 & Fragilaria sp. & 15,24 & - & - & - & - & - & - & - & - \\
\hline 89 & $\begin{array}{l}\text { Fragilaria ulna (Nitzsch) } \\
\text { L.-B. }\end{array}$ & $\begin{array}{l}4,5,8,11,13 \\
14,18-21,26 \\
27,29,33,34\end{array}$ & P-B & temp & st-str & es & $\beta$ & $\mathrm{i}$ & ind & $\mathrm{k}$ \\
\hline 90 & $\begin{array}{l}\text { Fragilaria vaucheriae (Kütz.) } \\
\text { B. Peters. }\end{array}$ & $\begin{array}{l}8,14,16,17, \\
18,20,21,27, \\
28,33,34\end{array}$ & $\mathrm{P}$ & - & - & - & - & $\mathrm{i}$ & alf & $\mathrm{Ha}$ \\
\hline
\end{tabular}


Tab. 2. - continued

\begin{tabular}{|c|c|c|c|c|c|c|c|c|c|c|}
\hline No & Taxon & No of Lake & Habitat & $\mathrm{T}$ & Reo & $\mathrm{D}$ & $\mathrm{S}$ & Hal & $\mathrm{pH}$ & Geo \\
\hline 91 & $\begin{array}{l}\text { Fragilariforma constricta } \\
\quad \text { (Ehrb.) Williams et Round }\end{array}$ & 14 & B & - & - & - & - & $\mathrm{i}$ & acf & $\mathrm{Ha}$ \\
\hline 92 & $\begin{array}{l}\text { Fragilariforma virescens } \\
\quad \text { (Ralfs) Williams et Round }\end{array}$ & 14,18 & P-B & - & st & es & $\mathrm{x}$ & $\mathrm{i}$ & ind & $\mathrm{k}$ \\
\hline 93 & $\begin{array}{l}\text { Fragilariopsis separanda } \\
\text { (Hust.) Hasle }\end{array}$ & 24 & - & - & - & - & - & hl & - & $\mathrm{Ha}$ \\
\hline 94 & Frustulia sp. & 24 & - & - & - & - & - & - & - & - \\
\hline 95 & $\begin{array}{l}\text { Geissleria schoenfeldii (Hust.) } \\
\text { L.-B. et Metzetlin in L.-B. }\end{array}$ & 20 & B & - & - & - & - & $\mathrm{i}$ & alf & $\mathrm{b}$ \\
\hline 96 & $\begin{array}{l}\text { Gomphonema acuminatum } \\
\text { Ehrb. var. acuminatum }\end{array}$ & 11,20 & P-B & - & st & es & $\beta$ & $\mathrm{i}$ & alf & $\mathrm{k}$ \\
\hline 97 & $\begin{array}{l}\text { Gomphonema acuminatum var. } \\
\text { coronatum (Ehrb.) W. Sm. }\end{array}$ & $14,18,20$ & P-B & - & st & - & $\beta$ & $\mathrm{i}$ & ind & $\mathrm{k}$ \\
\hline 98 & Gomphonema augur Ehrb. & 14,18 & B & - & - & es & - & $\mathrm{i}$ & ind & $\mathrm{k}$ \\
\hline 99 & Gomphonema clavatum Ehrb. & $8,18,33$ & B & - & - & es & - & $\mathrm{i}$ & - & $\mathrm{k}$ \\
\hline 100 & $\begin{array}{l}\text { Gomphonema parvulum } \\
\text { (Kütz.) Kütz. }\end{array}$ & $8,11,33$ & B & temp & str & es & $\beta$ & $\mathrm{i}$ & ind & $\mathrm{k}$ \\
\hline 101 & Gomphonema truncatum Ehrb. & $8,14,18,20$ & P-B & - & - & es & $\beta-\alpha$ & - & - & $\mathrm{k}$ \\
\hline 102 & $\begin{array}{l}\text { Gyrosigma acuminatum } \\
\text { (Kütz.) Rabenh. }\end{array}$ & $\begin{array}{l}5,8,18,22,26 \\
34\end{array}$ & B & cool & - & - & $\beta$ & $\mathrm{i}$ & alf & $\mathrm{k}$ \\
\hline 103 & $\begin{array}{l}\text { Gyrosigma spenceri (W. Sm.) } \\
\text { Cl. var. spenceri }\end{array}$ & $9,16,22,33$ & B & - & - & es & o & $\mathrm{mh}$ & alf & $\mathrm{k}$ \\
\hline 104 & $\begin{array}{l}\text { Gyrosigma spenceri var. } \\
\text { nodiferum Grun. }\end{array}$ & $3,9,13,14,28$ & B & - & - & - & - & $\mathrm{i}$ & ind & $\mathrm{b}$ \\
\hline 105 & $\begin{array}{l}\text { Hippodonta hungarica (Grun.) } \\
\text { L.-B., Metzeltin et Witkowski }\end{array}$ & $\begin{array}{l}14,16,18,20, \\
21,34\end{array}$ & B & - & st-str & es & $\beta$ & $\mathrm{i}$ & alf & $\mathrm{k}$ \\
\hline 106 & Luticola mutica (Kütz.) Mann & 33,34 & B & - & - & $\mathrm{sp}$ & $o-\beta$ & - & - & $\mathrm{k}$ \\
\hline 107 & Mastogloia sp. & $11,20,33$ & & - & - & - & - & - & - & - \\
\hline 108 & Melosira varians Ag. & 34 & P-B & temp & st-str & es & $\beta$ & hl & alf & $\mathrm{k}$ \\
\hline 109 & Navicula exigua Grun. & $\begin{array}{l}5,7-9,14,16 \\
20,22,25-28, \\
33,34\end{array}$ & B & - & - & es & & $\mathrm{i}$ & alf & $\mathrm{k}$ \\
\hline 110 & Navicula gregaria Donk. & 8 & B & - & - & es & $\beta$ & $\mathrm{mh}$ & alf & $\mathrm{k}$ \\
\hline 111 & Navicula peregrina (Ehrb.) Kütz. & 34 & B & - & - & es & o & $\mathrm{mh}$ & alf & $\mathrm{k}$ \\
\hline 112 & Navicula rhynchocephala Kütz. & $8,14,18,28$ & B & - & - & - & $\alpha$ & hl & alf & $\mathrm{k}$ \\
\hline 113 & Navicula sp. & 3,20 & - & - & - & - & - & - & - & - \\
\hline 114 & $\begin{array}{l}\text { Navicula viridula (Kütz.) } \\
\text { Ehrb. }\end{array}$ & $\begin{array}{l}5,8,14,18,20 \\
28,33,34\end{array}$ & B & - & - & es & $\alpha$ & $\mathrm{hl}$ & alf & $\mathrm{k}$ \\
\hline 115 & Neidium dubium (Ehrb.) Cl. & 10,20 & B & - & - & & $\beta$ & $\mathrm{i}$ & alf & $\mathrm{k}$ \\
\hline 116 & Neidium iridis (Ehrb.) Cl. & $8,18,20$ & $\mathrm{~B}$ & - & st & es & o & $\mathrm{hb}$ & ind & $\mathrm{k}$ \\
\hline 117 & $\begin{array}{l}\text { Nitzschia acicularis (Kütz.) } \\
\text { W. Sm. }\end{array}$ & $\begin{array}{l}2,5,9,14,15 \\
18,20-22,24 \\
27,28,33,34\end{array}$ & P-B & temp & - & es & $\alpha$ & $\mathrm{i}$ & alf & $\mathrm{k}$ \\
\hline
\end{tabular}


Tab. 2. - continued

\begin{tabular}{|c|c|c|c|c|c|c|c|c|c|c|}
\hline No & Taxon & No of Lake & Habitat & $\mathrm{T}$ & Reo & $\mathrm{D}$ & S & Hal & $\mathrm{pH}$ & Geo \\
\hline 118 & Nitzschia clausii Hantzsch & 11,29 & B & - & & es & $o-\alpha$ & $\mathrm{mh}$ & acf & $\mathrm{k}$ \\
\hline 119 & $\begin{array}{l}\text { Nitzschia dissipata (Kütz.) } \\
\text { Grun. }\end{array}$ & $\begin{array}{l}11,18,20,22, \\
25,34\end{array}$ & B & - & st-str & sx & $\beta$ & $\mathrm{i}$ & alf & $\mathrm{k}$ \\
\hline 120 & $\begin{array}{l}\text { Nitzschia filiformis (W. Sm.) } \\
\text { V. H. }\end{array}$ & $8,9,14,28,34$ & B & - & - & es & $\alpha-\beta$ & hl & - & $\mathrm{k}$ \\
\hline 121 & $\begin{array}{l}\text { Nitzschia linearis (C. Ag.) } \\
\text { W. Sm. }\end{array}$ & $\begin{array}{l}3,8,20,28,31, \\
34\end{array}$ & B & temp & - & es & $\beta$ & $\mathrm{i}$ & alf & $\mathrm{k}$ \\
\hline 122 & Nitzschia macilenta Greg. & $14,18,20,34$ & - & - & - & - & - & hl & - & - \\
\hline 123 & $\begin{array}{l}\text { Nitzschia palea (Kütz.) } \\
\text { W. Sm. }\end{array}$ & $\begin{array}{l}5,7,8,12-15 \\
18,20,21,27 \\
28,33,34\end{array}$ & P-B & temp & - & $\mathrm{sp}$ & $\beta-\alpha$ & $\mathrm{i}$ & ind & $\mathrm{k}$ \\
\hline 124 & Nitzschia reversa W. Sm. & 5 & - & - & - & - & - & hl & - & $\mathrm{k}$ \\
\hline 125 & $\begin{array}{l}\text { Nitzschia sigmoidea (Nitzsch) } \\
\text { W. Sm. }\end{array}$ & 20 & P-B & - & - & - & $\beta$ & $\mathrm{i}$ & alf & $\mathrm{k}$ \\
\hline 126 & Nitzschia sp. & 22,24 & - & - & - & - & - & - & - & - \\
\hline 127 & $\begin{array}{l}\text { Nitzschia vermicularis (Kütz.) } \\
\text { Hantzsch in Rabenh. }\end{array}$ & $3,5,11,12$ & B & - & - & - & $\beta$ & $\mathrm{i}$ & alf & $\mathrm{k}$ \\
\hline 128 & $\begin{array}{l}\text { Pinnularia gibba Ehrb. var. } \\
\quad \text { gibba }\end{array}$ & $18,20,33$ & B & - & - & es & - & $\mathrm{i}$ & ind & $\mathrm{b}$ \\
\hline 129 & $\begin{array}{l}\text { Pinnularia gibba var. } \\
\text { subundulata A. Mayer }\end{array}$ & 20,34 & B & - & - & - & - & $\mathrm{i}$ & - & $\mathrm{b}$ \\
\hline 130 & $\begin{array}{l}\text { Pinnularia microstauron } \\
\text { (Ehrb.) Cl. var. microstauron }\end{array}$ & 20,29 & B & temp & - & $\mathrm{sp}$ & o & $\mathrm{i}$ & ind & $\mathrm{k}$ \\
\hline 131 & $\begin{array}{l}\text { Pinnularia microstauron var. } \\
\text { brebissonii (Kütz.) Mayer }\end{array}$ & 8 & B & - & st-str & es & $\beta$ & $\mathrm{i}$ & ind & $\mathrm{k}$ \\
\hline 132 & $\begin{array}{l}\text { Pinnularia viridis (Nitzsch) } \\
\text { Ehrb. }\end{array}$ & $\begin{array}{l}3,14,20,22 \\
29,31,32,34\end{array}$ & P-B & temp & - & es & $\beta$ & $\mathrm{i}$ & ind & $\mathrm{k}$ \\
\hline 133 & Pinnularia sp. & 18 & B & - & - & - & - & - & - & - \\
\hline 134 & $\begin{array}{l}\text { Rhoicosphenia abbreviata } \\
\text { (C. Ag.) L.-B. }\end{array}$ & $\begin{array}{l}4,8,9,14,15 \\
17,27,28,33 \\
34\end{array}$ & P-B & - & - & es & $\beta$ & $\mathrm{i}$ & alf & $\mathrm{k}$ \\
\hline 135 & Rhopalodia gibba (Ehrb.) Müll. & $14,18,20,29$ & B & temp & - & es & o & $\mathrm{i}$ & alb & $\mathrm{k}$ \\
\hline 136 & $\begin{array}{l}\text { Rhopalodia musculus (Kütz.) } \\
\text { O. Müll. }\end{array}$ & 29 & P-B & - & - & & $\mathrm{x}$ & $\mathrm{mh}$ & alb & $\mathrm{k}$ \\
\hline 137 & $\begin{array}{l}\text { Sellaphora pupula (Kütz.) } \\
\text { Mereschkowsky }\end{array}$ & 18,34 & B & eterm & st & $\mathrm{sp}$ & $\alpha$ & hl & ind & $\mathrm{k}$ \\
\hline 138 & $\begin{array}{l}\text { Stauroneis anceps Ehrb. var. } \\
\text { anceps }\end{array}$ & $\begin{array}{l}6,8,9,11,16 \\
18,20,22,28 \\
33\end{array}$ & P-B & - & - & sx & $\beta$ & $\mathrm{i}$ & ind & $\mathrm{k}$ \\
\hline 139 & $\begin{array}{l}\text { Stauroneis anceps var. gracilis } \\
\text { Rabenh. }\end{array}$ & 8 & B & - & - & sx & - & $\mathrm{i}$ & ind & $\mathrm{k}$ \\
\hline 140 & $\begin{array}{l}\text { Stauroneis phoenicenteron } \\
\text { (Nitzsch) Ehrb. f. } \\
\text { phoenicenteron }\end{array}$ & 18,20 & B & temp & - & $\mathrm{s}$ & $\beta$ & $\mathrm{i}$ & ind & $\mathrm{k}$ \\
\hline
\end{tabular}


Tab. 2. - continued

\begin{tabular}{|c|c|c|c|c|c|c|c|c|c|c|}
\hline No & Taxon & No of Lake & Habitat & $\mathrm{T}$ & Reo & $\mathrm{D}$ & $\mathrm{S}$ & Hal & $\mathrm{pH}$ & Geo \\
\hline 141 & $\begin{array}{l}\text { Stauroneis phoenicenteron } \mathrm{f} . \\
\text { gracilis (Ehrb.) Hust. }\end{array}$ & 20 & - & - & - & - & - & - & ind & - \\
\hline 142 & Stauroneis sp. & 20 & B & - & - & - & - & - & - & - \\
\hline 143 & $\begin{array}{l}\text { Stephanodiscus hantzschii } \\
\text { Grun. }\end{array}$ & $\begin{array}{l}1,7-9,18,20 \\
33,34\end{array}$ & P-B & temp & st & es & $\alpha$ & $\mathrm{i}$ & alf & $\mathrm{k}$ \\
\hline 144 & Stephanodiscus sp. & 5 & - & - & - & - & & - & - & - \\
\hline 145 & $\begin{array}{l}\text { Surirella brebissonii Kram. } \\
\text { et L.-B. }\end{array}$ & $20,27,33$ & $\mathrm{~B}$ & - & st-str & & $\beta-\alpha$ & $\mathrm{i}$ & alf & $\mathrm{k}$ \\
\hline 146 & Surirella linearis W. Sm. & 14 & P-B & - & - & es & $\beta$ & $\mathrm{i}$ & ind & $\mathrm{Ha}$ \\
\hline 147 & Surirella ovalis Bréb. & $16,26,29,33$ & P-B & - & - & es & o & $\mathrm{mh}$ & alf & $\mathrm{k}$ \\
\hline 148 & $\begin{array}{l}\text { Surirella ovata var. pinnata } \\
\text { (W. Sm.) Rabenh. }\end{array}$ & $20,27,33,34$ & $\mathrm{~B}$ & - & - & es & $\beta$ & $\mathrm{i}$ & alf & $\mathrm{k}$ \\
\hline 149 & Surirella tenera Greg. & 34 & P-B & - & st & es & $\beta$ & $\mathrm{i}$ & alf & $\mathrm{k}$ \\
\hline 150 & Tryblionella gracilis W. Sm. & $5,17,20,23,29$ & $\mathrm{~B}$ & - & - & - & - & - & - & $\mathrm{k}$ \\
\hline 151 & $\begin{array}{l}\text { Tryblionella hungarica } \\
\text { (Grun.) D. G. Mann }\end{array}$ & - & P-B & - & - & $\mathrm{sp}$ & $\alpha$ & $\mathrm{mh}$ & alf & $\mathrm{k}$ \\
\hline \multicolumn{11}{|c|}{ Chlorophyta } \\
\hline 152 & Actinastrum hantzschii Lagerh. & $8,14,21,34$ & P-B & - & st-str & - & $\beta$ & $\mathrm{i}$ & - & $\mathrm{k}$ \\
\hline 153 & $\begin{array}{l}\text { Ankistrodesmus falcatus } \\
\text { (Corda) Ralfs }\end{array}$ & 20 & P-B & - & st-str & & $\beta$ & $\mathrm{hb}$ & - & $\mathrm{k}$ \\
\hline 154 & $\begin{array}{l}\text { Ankistrodesmus fusiformis } \\
\text { Corda sensu Korsch. }\end{array}$ & 14,20 & P-B & - & st-str & - & - & $\mathrm{i}$ & - & $\mathrm{k}$ \\
\hline 155 & $\begin{array}{l}\text { Binuclearia lauterbornii } \\
\text { (Schmidle) Pr.-Lavr. }\end{array}$ & 1,23 & $\mathrm{P}$ & - & - & - & - & - & - & $\mathrm{Ha}$ \\
\hline 156 & $\begin{array}{l}\text { Chaetophora pisiformis } \\
\text { (Roth) Ag. }\end{array}$ & 18,32 & B & - & st-str & - & - & - & - & $\mathrm{k}$ \\
\hline 157 & Chlamydomonas sp. & 20 & & - & & - & - & - & - & - \\
\hline 158 & $\begin{array}{l}\text { Cladophora fracta (Müll. ex } \\
\text { Vahl.) Kütz. }\end{array}$ & $\begin{array}{l}4,5,9,13,14 \\
22,27,28,33, \\
34\end{array}$ & P-B & - & st-str & - & $\beta$ & - & - & $\mathrm{k}$ \\
\hline 159 & $\begin{array}{l}\text { Cladophora glomerata (L.) } \\
\text { Kütz. }\end{array}$ & 11 & P-B & - & st-str & - & $\beta$ & $\mathrm{i}$ & alf & $\mathrm{k}$ \\
\hline 160 & $\begin{array}{l}\text { Closterium acerosum } \\
\text { (Schrank) Ehrb. }\end{array}$ & 28 & P-B & - & st-str & - & $\alpha$ & $\mathrm{i}$ & ind & $\mathrm{k}$ \\
\hline 161 & Closterium dianae Ehrb. & $\begin{array}{l}5,18,20,33 \\
34\end{array}$ & P-B & - & st-str & - & o & - & - & $\mathrm{k}$ \\
\hline 162 & $\begin{array}{l}\text { Closterium ehrenbergii } \\
\text { Menegh. }\end{array}$ & 15 & P-B & - & st-str & - & $\beta$ & $\mathrm{hb}$ & ind & $\mathrm{k}$ \\
\hline 163 & $\begin{array}{l}\text { Closterium gracile Bréb. in } \\
\text { Chevalier }\end{array}$ & 8,22 & P-B & - & st-str & - & - & $\mathrm{hb}$ & acf & $\mathrm{k}$ \\
\hline 164 & Closterium sp. & 8 & & - & - & - & - & - & - & - \\
\hline 165 & $\begin{array}{l}\text { Coelastrum microporum } \\
\text { Näg. in A. Br. }\end{array}$ & 8,33 & P-B & - & st-str & - & $\beta$ & $\mathrm{i}$ & ind & $\mathrm{k}$ \\
\hline
\end{tabular}


Tab. 2. - continued

\begin{tabular}{|c|c|c|c|c|c|c|c|c|c|c|}
\hline No & Taxon & No of Lake & Habitat & $\mathrm{T}$ & Reo & $\mathrm{D}$ & $\mathrm{S}$ & Hal & $\mathrm{pH}$ & Geo \\
\hline 166 & Coelastrum sphaericum Näg. & $8,14,20$ & P-B & - & st-str & - & - & $\mathrm{i}$ & - & $\mathrm{k}$ \\
\hline 167 & $\begin{array}{l}\text { Coenochloris pyrenoidosa } \\
\text { Korsch. }\end{array}$ & $11,14,18$ & $\mathrm{P}$ & - & - & - & - & hl & - & $\mathrm{Ha}$ \\
\hline 168 & $\begin{array}{l}\text { Coenococcus polycoccus } \\
\text { (Korsch.) Hind. }\end{array}$ & $\begin{array}{l}4,11,15,18 \\
20,33,34\end{array}$ & $\mathrm{P}$ & - & st & - & - & - & - & $\mathrm{k}$ \\
\hline 169 & $\begin{array}{l}\text { Coenocystis planktonica } \\
\text { Korsch. }\end{array}$ & 12 & $\mathrm{P}$ & - & st & - & $\beta$ & $\mathrm{i}$ & - & $\mathrm{k}$ \\
\hline 170 & $\begin{array}{l}\text { Coenocystis subcylindrica } \\
\text { Korsch. }\end{array}$ & 15,27 & $\mathrm{P}$ & - & - & - & - & $\mathrm{i}$ & - & $\mathrm{b}$ \\
\hline 171 & Cosmarium punctulatum Bréb. & $4,18,27$ & P-B & - & - & - & - & $\mathrm{hb}$ & acf & $\mathrm{k}$ \\
\hline 172 & Cosmarium sp. & $\begin{array}{l}4,14,18,27 \\
34\end{array}$ & & - & - & - & - & - & - & - \\
\hline 173 & $\begin{array}{l}\text { Crucigenia tetrapedia } \\
\quad \text { (Kirchn.) W. et G. S. West }\end{array}$ & $\begin{array}{l}4,8,20,27,33, \\
34\end{array}$ & P-B & - & st-str & - & $\beta$ & $\mathrm{i}$ & ind & $\mathrm{k}$ \\
\hline 174 & $\begin{array}{l}\text { Crucigeniella apiculata } \\
\text { (Lemm.) Komárek }\end{array}$ & 34 & P-B & - & st-str & - & - & - & - & $\mathrm{k}$ \\
\hline 175 & $\begin{array}{l}\text { Desmodesmus brasiliensis } \\
\text { (Bohlin) Hegew. }\end{array}$ & $8,20,33$ & P-B & - & st-str & - & $\beta$ & - & - & $\mathrm{k}$ \\
\hline 176 & $\begin{array}{l}\text { Desmodesmus denticulatus } \\
\text { (Lagerh.) An, Friedl et } \\
\text { Hegew. }\end{array}$ & 20 & P-B & - & st-str & - & $\beta$ & $\mathrm{i}$ & - & $\mathrm{k}$ \\
\hline 177 & $\begin{array}{l}\text { Desmodesmus opoliensis } \\
\text { (P. Richt.) Hegew. }\end{array}$ & 20 & P-B & - & st-str & - & - & - & - & $\mathrm{k}$ \\
\hline 178 & $\begin{array}{l}\text { Desmodesmus spinosus } \\
\text { (K. Biswas) Hegew. }\end{array}$ & 18 & P-B & - & st-str & - & o- $\beta$ & - & - & $\begin{array}{l}\mathrm{Ha}, \\
\mathrm{Nt}\end{array}$ \\
\hline 179 & $\begin{array}{l}\text { Dictyosphaerium pulchellum } \\
\text { Wood }\end{array}$ & $4,8,14,20,21$ & P-B & - & st-str & - & - & $\mathrm{i}$ & ind & $\mathrm{k}$ \\
\hline 180 & Elakatothrix acuta Pasch. & 20 & $\mathrm{P}$ & - & - & - & - & $\mathrm{i}$ & - & $\mathrm{k}$ \\
\hline 181 & Elakatothrix gelatinosa Wille & 20 & $\mathrm{P}$ & - & st-str & - & o & $\mathrm{i}$ & - & $\mathrm{k}$ \\
\hline 182 & $\begin{array}{l}\text { Eremosphera gigas } \\
\text { (W. Archer) Fott et Kalina }\end{array}$ & $8,15,34$ & $\mathrm{P}$ & - & - & - & - & $\mathrm{i}$ & acf & $\mathrm{k}$ \\
\hline 183 & Franceia tenuispina Korsch. & 18 & $\mathrm{P}$ & - & & - & - & - & - & $\mathrm{Ha}$ \\
\hline 184 & $\begin{array}{l}\text { Lagerheimia ciliata (Lagerh.) } \\
\text { Chod. }\end{array}$ & 20 & P-B & - & st-str & - & - & - & - & $\mathrm{k}$ \\
\hline 185 & Lagerheimia genevensis Chod. & 1,20 & $\mathrm{P}$ & - & & - & - & $\mathrm{i}$ & - & $\mathrm{k}$ \\
\hline 186 & Micrasterias sp. & 18 & & - & & - & - & - & - & - \\
\hline 187 & $\begin{array}{l}\text { Monoraphidium arcuatum } \\
\text { (Korsch.) Hind. }\end{array}$ & $1,20,28,34$ & P-B & - & st-str & - & - & - & - & $\mathrm{k}$ \\
\hline 188 & $\begin{array}{l}\text { Monoraphidium contortum } \\
\text { (Thur.) Kom.-Legn. }\end{array}$ & $\begin{array}{l}1,8,9,14,15 \\
18,20,21,27 \\
28,33\end{array}$ & P-B & - & st-str & - & - & - & - & $\mathrm{k}$ \\
\hline 189 & $\begin{array}{l}\text { Monoraphidium griffithii } \\
\text { (Berk.) Kom.-Legn. in Fott }\end{array}$ & $\begin{array}{l}7,8,9,14,18 \\
20,21,28,33 \\
34\end{array}$ & P-B & - & st-str & - & - & - & - & $\mathrm{k}$ \\
\hline
\end{tabular}


Tab. 2. - continued

\begin{tabular}{|c|c|c|c|c|c|c|c|c|c|c|}
\hline No & Taxon & No of Lake & Habitat & $\mathrm{T}$ & Reo & $\mathrm{D}$ & $S$ & Hal & $\mathrm{pH}$ & Geo \\
\hline 190 & $\begin{array}{l}\text { Monoraphidium komarkovae } \\
\text { Nyg. }\end{array}$ & 22 & $\mathrm{P}$ & - & st-str & - & - & - & - & $\mathrm{k}$ \\
\hline 191 & $\begin{array}{l}\text { Monoraphidium minutum } \\
\text { (Näg.) Kom.-Legn. }\end{array}$ & 1 & P-B & - & st-str & - & - & - & - & $\mathrm{k}$ \\
\hline 192 & Mougeotia sp. & $\begin{array}{l}11,18,20,28, \\
33\end{array}$ & & - & & - & - & - & - & - \\
\hline 193 & Nephrochlamys rotunda Korsch. & 20 & P-B & - & st-str & - & - & - & - & $\mathrm{Ha}$ \\
\hline 194 & Oedogonium sp. & $\begin{array}{l}3,11,14,15 \\
20,29-32\end{array}$ & & - & - & - & - & - & - & - \\
\hline 195 & Oocystis lacustris Chod. & 4 & P-B & - & st-str & - & $\beta$ & hl & - & $\mathrm{k}$ \\
\hline 196 & Oocystis submarina Lagerh. & $1,8,27,33$ & P-B & - & st & - & - & $\mathrm{i}$ & - & $\mathrm{k}$ \\
\hline 197 & Palmodictyon lobatum Korsch. & 15 & $\mathrm{~B}$ & - & st-str & - & - & - & - & $\mathrm{k}$ \\
\hline 198 & $\begin{array}{l}\text { Pediastrum boryanum (Turp.) } \\
\text { Menegh. }\end{array}$ & $\begin{array}{l}1,14,18,20 \\
27,33,34\end{array}$ & P-B & - & st-str & - & $\beta$ & $\mathrm{i}$ & ind & $\mathrm{k}$ \\
\hline 199 & Pediastrum duplex Meyen & $4,14,27$ & P-B & - & st-str & - & $\beta$ & $\mathrm{i}$ & ind & $\mathrm{k}$ \\
\hline 200 & $\begin{array}{l}\text { Pediastrum kawraiskyi } \\
\text { Schmidle }\end{array}$ & 4,8 & P-B & - & st-str & - & - & - & - & $\mathrm{Ha}$ \\
\hline 201 & Pediastrum tetras (Ehrb.) Ralfs & $14,20,34$ & P-B & - & st-str & - & $\beta$ & $\mathrm{i}$ & ind & $\mathrm{k}$ \\
\hline 202 & Penium sp. & 18 & & - & - & - & - & - & - & - \\
\hline 203 & Raphidocelis sigmoidea Hind. & 20 & $\mathrm{P}$ & - & st-str & - & - & - & - & $\mathrm{b}$, \\
\hline 204 & $\begin{array}{l}\text { Raphidocelis subcapitata } \\
\text { (Korsch.) Nyg. }\end{array}$ & 8,33 & P-B & - & st-str & - & - & - & - & $\begin{array}{l}\mathrm{Ha}, \\
\mathrm{Nt}\end{array}$ \\
\hline 205 & $\begin{array}{l}\text { Scenedesmus acuminatus } \\
\text { (Lagerh.) Chod. }\end{array}$ & $18,20,27$ & P-B & - & st-str & - & $\beta$ & $\mathrm{i}$ & ind & $\mathrm{k}$ \\
\hline 206 & $\begin{array}{l}\text { Scenedesmus acutiformis var. } \\
\text { costatus (Hub.-Pest.) Pankow }\end{array}$ & 18 & P-B & - & - & - & - & - & - & $\mathrm{k}$ \\
\hline 207 & Scenedesmus acutus Meyen & $14,18,20,28$ & P-B & - & st-str & - & $o-\beta$ & $\mathrm{i}$ & - & $\mathrm{k}$ \\
\hline 208 & $\begin{array}{l}\text { Scenedesmus apiculatus } \\
\text { (W. et G. S. West) Chod. } \\
\text { var. apiculatus }\end{array}$ & 18,28 & $\mathrm{P}$ & - & st-str & - & - & - & - & $\begin{array}{c}\mathrm{Ha}, \\
\mathrm{Pt}\end{array}$ \\
\hline 209 & $\begin{array}{l}\text { Scenedesmus apiculatus var. } \\
\text { indicus (Hortob.) Tzarenko }\end{array}$ & 20 & P-B & - & st-str & - & - & - & - & $\begin{array}{c}\mathrm{Ha}, \\
\mathrm{Nt}\end{array}$ \\
\hline 210 & $\begin{array}{l}\text { Scenedesmus arcuatus } \\
\text { (Lemm.) Lemm. }\end{array}$ & 14,20 & P-B & - & st-str & - & $\beta$ & $\mathrm{i}$ & - & $\mathrm{k}$ \\
\hline 211 & $\begin{array}{l}\text { Scenedesmus bijugatus } \\
\text { (Turp.) Kütz. }\end{array}$ & 27 & $\mathrm{P}$ & - & & - & - & $\mathrm{i}$ & ind & $\mathrm{k}$ \\
\hline 212 & $\begin{array}{l}\text { Scenedesmus disciformis } \\
\text { (Chod.) Fott. et Kom. }\end{array}$ & 20 & P-B & - & st-str & - & - & - & - & $\mathrm{k}$ \\
\hline 213 & Scenedesmus ellipticus Corda & $18,27,34$ & P-B & - & st-str & - & $0-\beta$ & - & - & $\mathrm{k}$ \\
\hline 214 & Scenedesmus gutwinskii Chod. & 20 & $\mathrm{P}$ & - & & - & - & - & - & $\mathrm{Ha}$ \\
\hline 215 & $\begin{array}{l}\text { Scenedesmus incrassatulus } \\
\text { Bohl. }\end{array}$ & 20 & P-B & - & st-str & - & - & - & - & $\mathrm{k}$ \\
\hline
\end{tabular}


Tab. 2. - continued

\begin{tabular}{|c|c|c|c|c|c|c|c|c|c|c|}
\hline No & Taxon & No of Lake & Habitat & $\mathrm{T}$ & Reo & $\mathrm{D}$ & $\mathrm{S}$ & Hal & $\mathrm{pH}$ & Geo \\
\hline 216 & $\begin{array}{l}\text { Scenedesmus obliquus (Turp.) } \\
\text { Kütz. }\end{array}$ & $1,8,11,20,33$ & P-B & - & st & - & - & $\mathrm{i}$ & - & $\mathrm{k}$ \\
\hline 217 & Scenedesmus obtusus Meyen & 30 & P-B & - & st-str & - & - & - & - & $\mathrm{Ha}$ \\
\hline 218 & $\begin{array}{l}\text { Scenedesmus quadricauda } \\
\text { (Turp.) Bréb. }\end{array}$ & $\begin{array}{l}5,14,18,20 \\
27,33,34\end{array}$ & $\mathrm{P}$ & - & - & - & - & $\mathrm{i}$ & ind & $\mathrm{k}$ \\
\hline 219 & $\begin{array}{l}\text { Schroederia setigera (Schrod.) } \\
\text { Lemm. }\end{array}$ & 8 & $\mathrm{P}$ & - & st-str & - & - & $\mathrm{i}$ & - & $\begin{array}{l}\mathrm{Ha}, \\
\mathrm{Nt}\end{array}$ \\
\hline 220 & Selenastrum gracile Reinsch & 14,20 & P-B & - & st-str & - & $\beta$ & - & - & $\mathrm{k}$ \\
\hline 221 & Spirogyra sp. & $\begin{array}{l}4,15,18 \\
27-30,33\end{array}$ & - & - & - & - & - & - & - & - \\
\hline 222 & Spirogyra weberi Kütz. & 29 & - & - & - & - & - & - & - & $\mathrm{k}$ \\
\hline 223 & Staurastrum gracile Ralfs & 18,27 & $\mathrm{P}$ & - & st & - & - & $\mathrm{i}$ & - & $\mathrm{k}$ \\
\hline 224 & Staurastrum sebaldii Reinsch. & 4 & P-B & - & - & - & - & - & acf & $\mathrm{k}$ \\
\hline 225 & Staurastrum sp. & 18 & - & - & - & - & - & - & - & - \\
\hline 226 & Staurodesmus sp. & 14 & - & - & - & - & - & - & - & - \\
\hline 227 & $\begin{array}{l}\text { Stigeoclonium tenue (Ag.) } \\
\text { Kütz. emend. Cox et Bold }\end{array}$ & 20 & B & - & st-str & - & $\alpha$ & - & - & $\mathrm{k}$ \\
\hline 228 & $\begin{array}{l}\text { Tetrachlorella alternans } \\
\text { (G. M. Smith) Korsch. }\end{array}$ & 14 & P-B & - & & - & - & - & - & $\mathrm{Ha}$ \\
\hline 229 & $\begin{array}{l}\text { Tetraedron caudatum (Corda) } \\
\text { Hansg. }\end{array}$ & 34 & P-B & - & st-str & - & $\beta$ & $\mathrm{i}$ & ind & $\mathrm{k}$ \\
\hline 230 & $\begin{array}{l}\text { Tetraedron incus (Teil.) } \\
\text { G. M. Smith }\end{array}$ & 18,20 & P-B & - & st-str & - & - & $\mathrm{i}$ & - & $\mathrm{k}$ \\
\hline 231 & $\begin{array}{l}\text { Tetraedron minimum (A. Br.) } \\
\text { Hansg. }\end{array}$ & $\begin{array}{l}1,18,20,27 \\
34\end{array}$ & P-B & - & st-str & - & $\beta$ & $\mathrm{i}$ & - & $\mathrm{k}$ \\
\hline 232 & Tetrastrum elegans Playf. & $8,20,33$ & $\mathrm{P}$ & - & st-str & - & - & $\mathrm{i}$ & - & $\mathrm{k}$ \\
\hline 233 & $\begin{array}{l}\text { Tetrastrum triacanthum } \\
\text { Korsch. }\end{array}$ & 20 & $\mathrm{P}$ & - & st-str & - & - & - & - & $\mathrm{Ha}$ \\
\hline 234 & Ulothrix tenerrima Kütz. & 29 & $\mathrm{~B}$ & - & - & - & - & $\mathrm{i}$ & - & $\mathrm{k}$ \\
\hline 235 & $\begin{array}{l}\text { Ulothrix zonata (Weber et } \\
\text { Mohr) Kütz. }\end{array}$ & $13,22,27,34$ & P-B & - & st-str & - & o & $\mathrm{i}$ & ind & $\mathrm{k}$ \\
\hline 236 & Ulothrix sp. & 27 & B & - & - & - & - & - & - & - \\
\hline 237 & Volvox aureus Ehrb. & 20 & $\mathrm{P}$ & - & st & - & $\beta$ & $\mathrm{i}$ & - & $\mathrm{k}$ \\
\hline 238 & Zygnema sp. & 18 & $\mathrm{~B}$ & - & - & - & - & - & - & - \\
\hline \multicolumn{11}{|c|}{ Chrysophyta } \\
\hline 239 & Dinobryon sertularia Ehrb. & 11 & $\mathrm{P}$ & - & - & - & - & $\mathrm{i}$ & - & $\mathrm{k}$ \\
\hline \multicolumn{11}{|c|}{ Cryptophyta } \\
\hline 240 & Cryptomonas sp. & $\begin{array}{l}1,9,16,18 \\
20-22,27,32\end{array}$ & $\mathrm{P}$ & - & - & - & - & - & - & - \\
\hline \multicolumn{11}{|c|}{ Dinophyta } \\
\hline 241 & $\begin{array}{l}\text { Glenodinium quadridens } \\
\text { (Stenis) Schiller. }\end{array}$ & 20 & $\mathrm{P}$ & - & - & - & - & - & - & $\mathrm{k}$ \\
\hline
\end{tabular}


Tab. 2. - continued

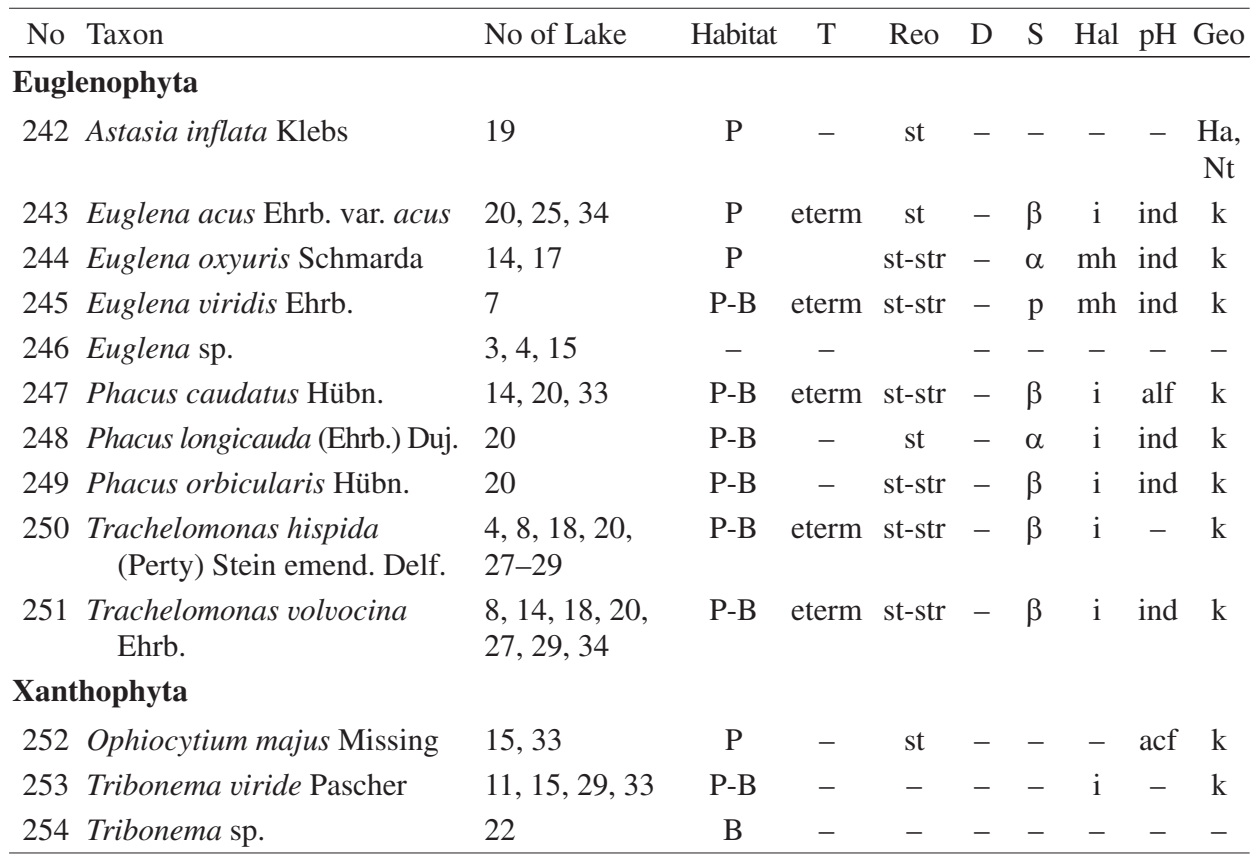

The lacustrine algoflora mainly consists of geographically widespread species (85\% cosmopolitan, $9 \%$ of pan-Holarctic distribution, and $4.5 \%$ of Boreal distribution (Tab. 2).

In the lakes of arid regions, algae occur over the water column and on hard substrates, with some preference for the latter (Fig. 2b). The most abundant periphyton species are cyanoprokaryotic Anabaena flos-aquae f. flos-aquae (Kamyshovoe Lake), Coelosphaerium minutissimum (Kulagul Lake), Phormidium retzii (Lake Great Kak), diatoms Amphora pediculus (Great Kak Lake), and green algae Binuclearia lauterbornii (Aike Lake) and Spirogyra weberi (Jarsor Stream)

With respect to $\mathrm{pH}$, the indicator species (HUSTEDT 1938-1939) are segregated into four groups among which the alkaliphiles prevail (Fig. 2c). Such distribution is characteristic of slightly alkalic conditions (Tab. 1). The most common alkaliphiles are Chroococcus turgidus, (Cyanoprokaryota) and Achnanthes minutissima, Amphora ovalis, A. pediculus, Caloneis amphisbaena, Epithemia turgida, Fragilaria vaucheriae, Navicula exigua, Nitzschia acicularis, N. palea, Rhoicosphenia abbreviata (Bacillariophyta). The diversity of $\mathrm{pH}$ indicators reflects the great amplitude of this variable.

Salinity indicators (HusteDT 1957) are assigned to five ecological groups (Fig. 2d), with oligohalobes-indifferents as a dominant group, although the oligohalobes-halophiles and mesohalobes are also common, as well as a single species of polyhalobes (Tab. 2). Among the oligohalobes-indifferents the most common are Amphora ovalis, Epithemia turgida, Fragilaria ulna, F. vaucheriae, Nitzschia acicularis, N. palea, Rhoicosphenia abbreviata (Bacillariophyta), Crucigenia tetrapedia, Pediastrum boryanum (Chlorophyta), Trachelomonas hispida, T. volvocina (Euglenophyta). Remarkably, the blue-greens are 

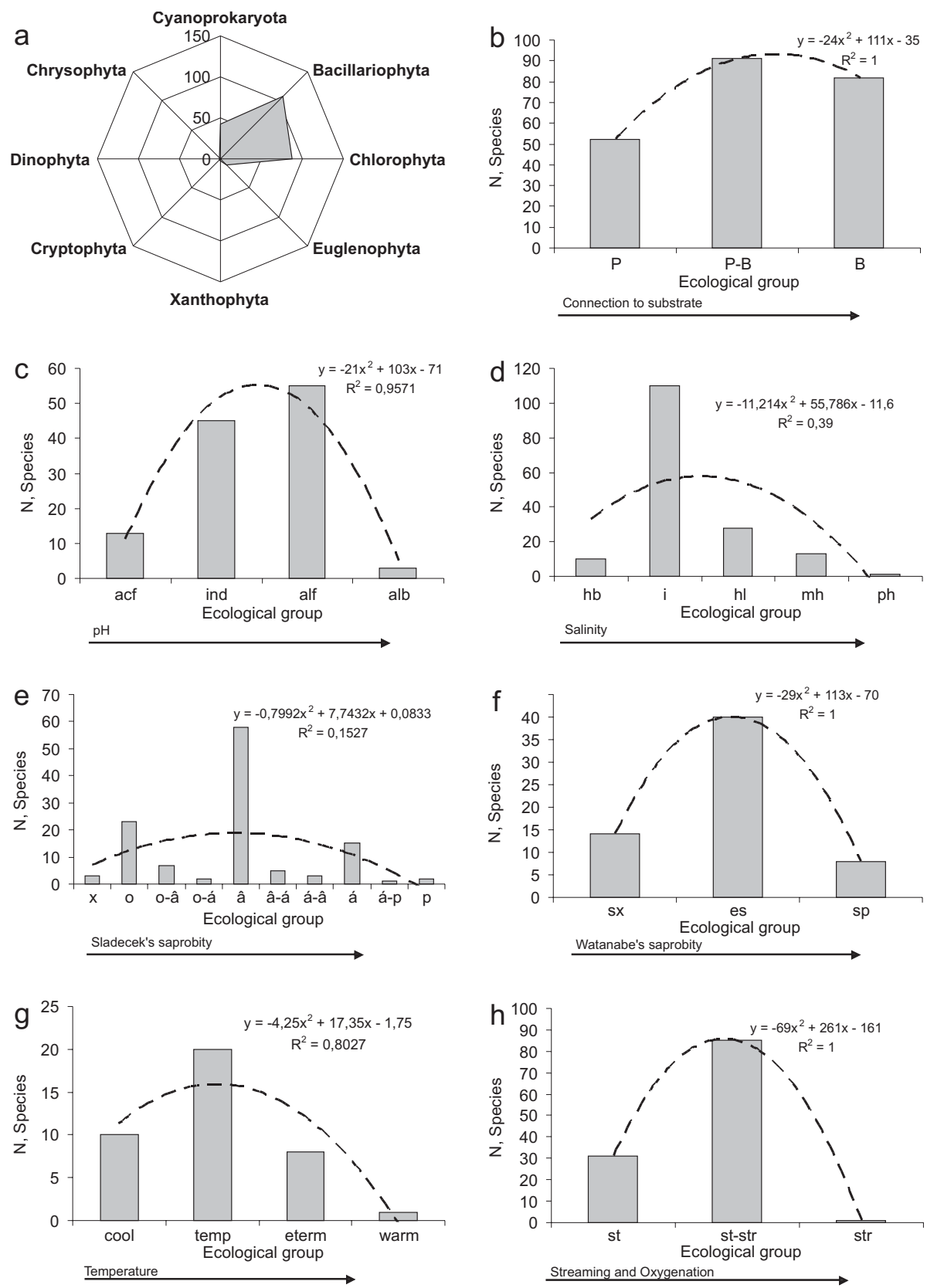

Fig. 2. Algal species diversity and ecology in the wetland lakes of Kazakhstan: a - distribution of species richness per taxonomic divisions; $b$ - distribution of species per habitat ecological groups; $\mathrm{c}$ - distribution of species per groups of $\mathrm{pH}$ indicators; $\mathrm{d}$ - distribution of species per groups of salinity indicators; e - distribution of species per groups of saprobity indicators (after SLÁDEČEK 1973); f - distribution of species per groups of saprobity indicators (after WATANABE 1986); $\mathrm{g}$ - distribution of species per groups of temperature indicators; $\mathrm{h}$ - distribution of species per groups of streaming and oxygenation indicators. 
mostly halophilic, but include also the only palyhalobic species Phormidium paulsenianum. All the halophobes are diatoms, among them several species of Eunotia.

Algal indicators of organic pollution are assigned to nine ecological groups (Figs. 2e, f; Tab. 2), representing the entire spectrum of indication systems (SLÁDEČEK 1973, 1986; WatANABE et al. 1986). Beta-mesosaprobionts prevail with Phormidium autumnale, Microcystis aeruginosa (Cyanoprokaryota), Fragilaria vaucheriae, Surirella ovata, Pinnularia

Tab. 3. Chemical variables (with standard deviation) and water quality classification based on concentrations of nitrate- nitrogen and phosphorus in the wetland lakes during 1-20 October 1999.

\begin{tabular}{|c|c|c|c|c|}
\hline \multirow[t]{2}{*}{ Lake } & \multirow[t]{2}{*}{$\mathrm{N}-\mathrm{NO}_{3}\left(\mathrm{mg} \mathrm{L}^{-1}\right)$} & \multirow[t]{2}{*}{$\mathrm{P}-\mathrm{PO}_{4}{ }^{3-}\left(\mathrm{mg} \mathrm{L}^{-1}\right)$} & \multicolumn{2}{|c|}{ Rank of water quality } \\
\hline & & & $\mathrm{N}-\mathrm{NO}_{3}$ & $\mathrm{P}-\mathrm{PO}_{4}{ }^{3-}$ \\
\hline Aike & $3.4 \pm 0.14$ & $0.16 \pm 0.01$ & $5 \mathrm{a}$ & $4 a$ \\
\hline Kulykol & $1.5 \pm 0.1$ & $0.06 \pm 0.00$ & $3 b$ & $3 b$ \\
\hline Kulykol (N2) & $1.1 \pm 0.0$ & $0.05 \pm 0.00$ & $3 b$ & $3 a$ \\
\hline Kulykol, kordon Jailma & $2.1 \pm 0.1$ & $0.30 \pm 0.01$ & $3 b$ & $4 b$ \\
\hline Kulykol, well Jailma & $1.0 \pm 0.0$ & $0.06 \pm 0.00$ & $3 \mathrm{a}$ & $3 b$ \\
\hline Stream Jarsor & $1.0 \pm 0.0$ & $0.01 \pm 0.00$ & $3 \mathrm{a}$ & $2 \mathrm{a}$ \\
\hline Jarsor & $8.6 \pm 0.3$ & $4.06 \pm 0.12$ & $5 b$ & $5 b$ \\
\hline Batpakkol & $2.3 \pm 0.1$ & $0.26 \pm 0.01$ & $4 \mathrm{~b}$ & $4 b$ \\
\hline Kulagul & $0.0 \pm 0.0$ & $0.98 \pm 0.03$ & 1 & $5 b$ \\
\hline Sankebay & $5.4 \pm 0.2$ & $0.54 \pm 0.20$ & $5 \mathrm{~b}$ & $5 \mathrm{a}$ \\
\hline Jarkol, Naurzum Natural Reserves* & $4.1 \pm 0.2$ & $1.08 \pm 0.03$ & $5 b$ & $5 b$ \\
\hline Kushmurun, south part & $3.5 \pm 0.1$ & $0.10 \pm 0.00$ & $5 \mathrm{a}$ & $3 b$ \\
\hline Kojbagor, coast & $2.0 \pm 0.1$ & $0.13 \pm 0.00$ & $4 a$ & $4 \mathrm{a}$ \\
\hline Kojbagor & $1.9 \pm 0.1$ & $0.27 \pm 0.01$ & $4 a$ & $4 b$ \\
\hline Tuntugur & $1.9 \pm 0.1$ & $0.12 \pm 0.00$ & $4 a$ & $4 b$ \\
\hline Tuntugur, coast & $2.0 \pm 0.1$ & $0.26 \pm 0.01$ & $4 a$ & $4 b$ \\
\hline River Karasu, near L. Tuntugur & $1.4 \pm 0.1$ & $0.23 \pm 0.01$ & $3 b$ & $4 b$ \\
\hline Bozshakol & $2.7 \pm 0.1$ & $0.20 \pm 0.01$ & $5 \mathrm{a}$ & $4 \mathrm{a}$ \\
\hline Bozshakol, north-east part & $8.5 \pm 0.3$ & $2.25 \pm 0.07$ & $5 b$ & $5 b$ \\
\hline Biesoygan & $2.1 \pm 0.1$ & $0.39 \pm 0.01$ & $4 b$ & $5 \mathrm{a}$ \\
\hline Sarykol & $1.5 \pm 0.1$ & $0.06 \pm 0.00$ & $3 b$ & $3 b$ \\
\hline Taly & $1.7 \pm 0.1$ & $0.15 \pm 0.00$ & $4 a$ & $4 a$ \\
\hline Kamyshovoe & $1.9 \pm 0.1$ & $0.11 \pm 0.00$ & $4 a$ & $4 a$ \\
\hline Jaman & $1.6 \pm 0.1$ & $0.08 \pm 0.00$ & $4 a$ & $3 b$ \\
\hline Shoshkaly & $1.3 \pm 0.1$ & $0.32 \pm 0.01$ & $3 b$ & $5 \mathrm{a}$ \\
\hline Annovskoe & $2.0 \pm 0.1$ & $0.01 \pm 0.00$ & $4 a$ & $2 \mathrm{a}$ \\
\hline Maybalyk & $1.4 \pm 0.1$ & $0.02 \pm 0.00$ & $3 b$ & $2 b$ \\
\hline Tahtakul & $1.8 \pm 0.1$ & $0.48 \pm 0.01$ & $4 a$ & $5 \mathrm{a}$ \\
\hline Sarybalyk & $1.8 \pm 0.1$ & $0.03 \pm 0.00$ & $4 a$ & $2 b$ \\
\hline Aksuat & $1.0 \pm 0.0$ & $0.01 \pm 0.00$ & $3 b$ & $2 \mathrm{a}$ \\
\hline Gr. Karakamys & $1.2 \pm 0.0$ & $0.01 \pm 0.00$ & $3 b$ & $2 \mathrm{a}$ \\
\hline
\end{tabular}

Note: the assessment of $\beta+\gamma$ radioactive pollution of water samples with detector-indicator of radioactivity QUARTEX RD 8901 shows that its level does not exceed $20 \mathrm{mkR} / \mathrm{h} . *^{*}$ - Naurzum Biosphere Reserve. 
viridis (Bacillariophyta), Cladophora, Crucigenia tetrapedia (Chlorophyta), Trachelomonas volvocina (Euglenophyta).

Indicators of temperature conditions reflect a wide range of temperature fluctuations. A group of temperate species prevails, but species of cold and warm waters are also present (Fig. 2g).

Among indicators of streaming and oxygenation, species of slightly turbulent waters moderate oxygenation prevail, yet in figure $2 \mathrm{~h}$, the summit of the trend is displaced toward the indicators of streaming highly oxygenized waters.

Tab. 4. Saprobity indices, species richness and classification of water quality in the wetland lakes of Kazakhstan during October 1999.

\begin{tabular}{lccccc}
\hline Lake & $\begin{array}{c}\text { No. of } \\
\text { algal } \\
\text { species }\end{array}$ & $\begin{array}{c}\text { Index of } \\
\text { saprobity } \\
(\mathrm{S})\end{array}$ & $\begin{array}{c}\text { Rank of water } \\
\text { quality based } \\
\text { on biological } \\
\text { variables }\end{array}$ & $\begin{array}{c}\text { Rank of water } \\
\text { quality based } \\
\text { on chemical } \\
\text { variables }\end{array}$ & $\begin{array}{c}\text { Water } \\
\text { Ecosystem } \\
\text { State Index } \\
\text { (WESI) }\end{array}$ \\
\hline Aike & 20 & 1.98 & $3 \mathrm{a}$ & $5 \mathrm{a}$ & 0.5 \\
Kulykol & 2 & 2.3 & $3 \mathrm{~b}$ & $3 \mathrm{~b}$ & 1.0 \\
Kulykol (N2) & 2 & 1.85 & $3 \mathrm{a}$ & $3 \mathrm{~b}$ & 0.8 \\
Kulykol, kordon Jailma & 3 & 2.3 & $3 \mathrm{~b}$ & $4 \mathrm{~b}$ & 0.7 \\
Kulykol, well Jailma & 3 & 2.35 & $3 \mathrm{~b}$ & $3 \mathrm{~b}$ & 1.0 \\
Stream Jarsor & 7 & 1.99 & $3 \mathrm{a}$ & $3 \mathrm{a}$ & 1.0 \\
Jarsor & - & - & - & $5 \mathrm{~b}$ & - \\
Batpakkol & 2 & 2.0 & $3 \mathrm{a}$ & $4 \mathrm{~b}$ & 0.6 \\
Kulagul & 4 & 2.38 & $3 \mathrm{~b}$ & $5 \mathrm{~b}$ & 0.5 \\
Sankebay & 5 & 1.93 & $3 \mathrm{a}$ & $5 \mathrm{~b}$ & 0.4 \\
Jarkol. Naurzum Natural Reserves & 2 & 1.7 & $3 \mathrm{a}$ & $5 \mathrm{~b}$ & 0.4 \\
Kushmurun, south part & 9 & 2.08 & $3 \mathrm{~b}$ & $4 \mathrm{a}$ & 0.8 \\
Kojbagor, coast & 29 & 2.03 & $3 \mathrm{~b}$ & $4 \mathrm{~b}$ & 0.7 \\
Kojbagor & 26 & 1.95 & $3 \mathrm{a}$ & $4 \mathrm{a}$ & 0.6 \\
Tuntugur & 6 & 1.93 & $3 \mathrm{a}$ & $4 \mathrm{~b}$ & 0.6 \\
Tuntugur, coast & 26 & 1.88 & $3 \mathrm{a}$ & $4 \mathrm{a}$ & 0.6 \\
Karasu River near L. Tuntugur & 2 & 1.47 & $2 \mathrm{~b}$ & $4 \mathrm{~b}$ & 0.7 \\
Bozshakol & 23 & 1.99 & $3 \mathrm{a}$ & $5 \mathrm{a}$ & 0.5 \\
Bozshakol, north-east part & 31 & 1.95 & $3 \mathrm{a}$ & $5 \mathrm{~b}$ & 0.4 \\
Biesoygan & 7 & 2.55 & $4 \mathrm{a}$ & $5 \mathrm{a}$ & 0.7 \\
Sarykol & 10 & 2.15 & $3 \mathrm{~b}$ & $3 \mathrm{~b}$ & 1.0 \\
Taly & 6 & 2.05 & $3 \mathrm{~b}$ & $4 \mathrm{a}$ & 0.8 \\
Kamyshovoe & 22 & 1.9 & $3 \mathrm{a}$ & $4 \mathrm{a}$ & 0.6 \\
Jaman & 2 & 1.78 & $3 \mathrm{a}$ & $4 \mathrm{a}$ & 0.6 \\
Shoshkaly & 2.53 & $4 \mathrm{a}$ & $5 \mathrm{a}$ & 0.7 \\
Annovskoe & - & - & $3 \mathrm{a}$ & $4 \mathrm{a}$ & 0.6 \\
Maybalyk & - & 1.71 & - & $3 \mathrm{~b}$ & - \\
Tahtakul & - & - & - & $5 \mathrm{~b}$ & - \\
Sarybalyk & 2 & 2.7 & $4 \mathrm{a}$ & $4 \mathrm{a}$ & - \\
Aksuat & - & - & - & $3 \mathrm{~b}$ & - \\
Karakamys & - & - & - & & \\
\hline
\end{tabular}




\section{Assessment of wetland lacustrine ecosystems according to the hydrochemical and hydrobiological variables}

Hydrochemical data for autumn of 1999 (dry period) are represented in tables 1 and 3. The analysis of water conductivity and mineralization reveals a group of highly mineralized lakes - Jarsor, Sankebay and Kushmurun - with salinity level above 7. The other lakes are brackish or freshwater. Practically all the investigated lakes show the neutral or slightly alkalic reaction typical of natural water bodies with active self-purification processes. Sul-

Tab. 5. Chemical variables (with standard deviation) and water quality classification based on concentrations of nitrate nitrogen and phosphorus in the wetland lakes during May-June 2000.

\begin{tabular}{|c|c|c|c|c|}
\hline \multirow[t]{2}{*}{ Lake } & \multirow[t]{2}{*}{$\mathrm{N}-\mathrm{NO}_{3}\left(\mathrm{mg} \mathrm{L}^{-1}\right)$} & \multirow[t]{2}{*}{$\mathrm{P}-\mathrm{PO}_{4}^{3}\left(\mathrm{mg} \mathrm{L}^{-1}\right)$} & \multicolumn{2}{|c|}{ Rank of water quality } \\
\hline & & & $\mathrm{N}-\mathrm{NO}_{3}$ & $\mathrm{P}_{-} \mathrm{PO}_{4}{ }^{3-}$ \\
\hline Alpash & $1.0 \pm 0.0$ & 0.01 & $3 a$ & $2 \mathrm{a}$ \\
\hline Kulykol & $1.2 \pm 0.0$ & 0.03 & $3 b$ & $2 b$ \\
\hline Gr. Kak & $2.2 \pm 0.1$ & 0.05 & $4 \mathrm{~b}$ & $3 a$ \\
\hline Suly & $1.4 \pm 0.1$ & 1.77 & $3 b$ & $5 b$ \\
\hline Balykty & $1.1 \pm 0.0$ & 0.02 & $3 b$ & $2 b$ \\
\hline Stream Jarsor & $1.0 \pm 0.0$ & 0.01 & $3 a$ & $2 \mathrm{a}$ \\
\hline Jarsor & $2.9 \pm 0.1$ & 0.32 & $5 \mathrm{a}$ & $5 \mathrm{a}$ \\
\hline Maybalyk & $2.2 \pm 0.1$ & 0.00 & $4 \mathrm{~b}$ & 1 \\
\hline Kulagul & $0.6 \pm 0.0$ & 0.17 & $3 a$ & $4 a$ \\
\hline Gr. Sankebay & $1.6 \pm 0.1$ & 0.09 & $4 a$ & $3 b$ \\
\hline Jarkol, Naurzum Natural Reserves* & $2.3 \pm 0.1$ & 0.17 & $4 \mathrm{~b}$ & $4 \mathrm{a}$ \\
\hline Chushkaly, Naurzum Natural Reserves* & $1.7 \pm 0.1$ & 0.28 & $4 a$ & $4 b$ \\
\hline Tounsor (Teniz) & $1.5 \pm 0.1$ & 0.00 & $3 b$ & 1 \\
\hline Kojbagor & $1.3 \pm 0.1$ & 0.07 & $3 b$ & $3 b$ \\
\hline Tuntugur & $1.5 \pm 0.1$ & 0.01 & $3 b$ & $2 \mathrm{a}$ \\
\hline Teniz & $1.0 \pm 0.0$ & 0.04 & $3 a$ & $3 \mathrm{a}$ \\
\hline Sultan & $0.9 \pm 0.0$ & 0.00 & $3 a$ & 1 \\
\hline Bozshakol & $1.7 \pm 0.1$ & 0.00 & $4 a$ & 1 \\
\hline Jarken & $0.9 \pm 0.0$ & 0.03 & $3 a$ & $2 b$ \\
\hline Jaltyr & $1.0 \pm 0.0$ & 1.00 & $3 a$ & $5 b$ \\
\hline Sarykol & $0.9 \pm 0.0$ & 0.00 & $3 a$ & 1 \\
\hline Jilandy & $1.2 \pm 0.0$ & 0.68 & $3 b$ & $5 b$ \\
\hline Kamyshovoe & $1.2 \pm 0.0$ & 0.02 & $3 b$ & $2 b$ \\
\hline Jaman & $1.2 \pm 0.0$ & 0.03 & $3 b$ & $2 b$ \\
\hline Shoshkaly, western part & $1.1 \pm 0.0$ & 0.03 & $3 b$ & $2 b$ \\
\hline Annovskoe & $1.2 \pm 0.0$ & 0.00 & $3 b$ & 1 \\
\hline Gr. Karakamys & $0.8 \pm 0.0$ & 0.04 & $3 a$ & $3 a$ \\
\hline Tahtakul & $1.1 \pm 0.0$ & 0.03 & $3 b$ & $2 b$ \\
\hline Sarybalyk & $1.5 \pm 0.1$ & 0.00 & $3 b$ & 1 \\
\hline Aksuat & $1.4 \pm 0.1$ & 0.02 & $3 b$ & $2 b$ \\
\hline Karakamys & $1.0 \pm 0.0$ & 0.68 & $3 a$ & $5 b$ \\
\hline
\end{tabular}


phide $\left(\mathrm{H}_{2} \mathrm{~S}\right.$ plus the acid-soluble sulfides of metals) concentration $2.005 \mathrm{mg} \mathrm{L}^{-1}$ was found in the northeastern part of Bozshakol Lake only, which is evidence of periodic anoxia.

The saprobity index $\mathrm{S}$ varies from 1.47 to 2.70 , which corresponds to $2 \mathrm{~b}-4 \mathrm{a}$ ranges of water quality (Tab. 4). The biotic component of lake ecosystems provides for a high level of self-purification.

Tab. 6. Saprobity index S, species richness and water quality classification in wetland lakes of Northern Kazakhstan in May-June 2000.

\begin{tabular}{|c|c|c|c|c|c|}
\hline Lake & $\begin{array}{c}\text { Maximum } \\
\text { no. of algal } \\
\text { species, } \\
\text { (per sample) }\end{array}$ & $\begin{array}{l}\text { Index of } \\
\text { saprobity } \\
\text { (S) }\end{array}$ & $\begin{array}{c}\text { Rank of } \\
\text { water quality } \\
\text { based on the } \\
\text { biological } \\
\text { variables }\end{array}$ & $\begin{array}{c}\text { Rank of } \\
\text { water quality } \\
\text { based on the } \\
\text { chemical } \\
\text { variables }\end{array}$ & $\begin{array}{c}\text { Water } \\
\text { Ecosystem } \\
\text { State Index } \\
\text { (WESI) }\end{array}$ \\
\hline Alpash & 14 & 1.74 & $3 a$ & $3 a$ & 1.0 \\
\hline Kulykol & 8 & 2.04 & $3 b$ & $3 b$ & 1.0 \\
\hline Gr. Kak & 9 & 2.11 & $3 b$ & $4 \mathrm{~b}$ & 0.7 \\
\hline Suly & 7 & 1.84 & $3 a$ & $4 \mathrm{~b}$ & 0.6 \\
\hline Balykty & 26 & 2.21 & $3 b$ & $3 b$ & 1.0 \\
\hline Stream Jarsor & 10 & 1.76 & $3 a$ & $3 a$ & 1.0 \\
\hline Jarsor & - & - & - & $5 \mathrm{a}$ & - \\
\hline Maybalyk & 4 & 2.04 & $3 b$ & $4 \mathrm{~b}$ & 0.7 \\
\hline Kulagul & 13 & 2.44 & $4 \mathrm{a}$ & $4 a$ & 1.0 \\
\hline Gr. Sankebay & 2 & 2.49 & $4 a$ & $4 \mathrm{a}$ & 1.0 \\
\hline $\begin{array}{l}\text { Jarkol, Naurzum Natural } \\
\text { Reserves }\end{array}$ & 6 & 1.96 & $3 a$ & $4 \mathrm{~b}$ & 0.6 \\
\hline $\begin{array}{l}\text { Chushkaly, Naurzum } \\
\text { Natural Reserves }\end{array}$ & 12 & 1.97 & $3 b$ & $4 \mathrm{~b}$ & 0.7 \\
\hline Tounsor (Teniz) & 30 & 1.87 & $3 a$ & $3 b$ & 0.8 \\
\hline Kojbagor & 64 & 2.04 & $3 b$ & $3 b$ & 1.0 \\
\hline Tuntugur & 23 & 2.12 & $3 b$ & $3 b$ & 1.0 \\
\hline Teniz & 25 & 1.96 & $3 \mathrm{a}$ & $3 a$ & 1.0 \\
\hline Sultan & 5 & 2.15 & $3 b$ & $3 a$ & 1.25 \\
\hline Bozshakol & 30 & 2.13 & $3 b$ & $4 \mathrm{a}$ & 0.8 \\
\hline Jarken & 23 & 2.00 & $3 b$ & $3 a$ & 1.25 \\
\hline Jaltyr & 6 & 1.86 & $3 a$ & $5 b$ & 0.4 \\
\hline Sarykol & 23 & 1.71 & $3 a$ & $3 a$ & 1.0 \\
\hline Jilandy & 6 & 2.05 & $3 b$ & $5 b$ & 0.5 \\
\hline Kamyshovoe & 53 & 1.86 & $3 b$ & $3 b$ & 1.0 \\
\hline Jaman & 40 & 2.10 & $3 b$ & $3 b$ & 1.0 \\
\hline Shoshkaly, western part & 23 & 1.87 & $3 b$ & $3 b$ & 1.0 \\
\hline Annovskoe & 10 & 1.81 & $3 a$ & $3 b$ & 0.8 \\
\hline Gr. Karakamys & 6 & 1.65 & $3 \mathrm{a}$ & $3 a$ & 1.0 \\
\hline Tahtakul & 4 & 1.80 & $3 a$ & $3 b$ & 0.8 \\
\hline Sarybalyk & 5 & 1.48 & $2 b$ & $3 b$ & 0.6 \\
\hline Aksuat & 1 & 2.70 & $4 a$ & $3 b$ & 1.2 \\
\hline Karakamys & 18 & 1.80 & $3 a$ & $5 \mathrm{~b}$ & 0.4 \\
\hline
\end{tabular}


In spring, the lakes Kak, Jarsor, Sankebay and Sarybalyk were strongly mineralized, with salinity above 7 (Tabs. 5, 6). The other lakes remained freshwater or brackish. The pH reaction was neutral or slightly acidic in all the lakes, characteristic of natural waters with active self-purification processes.

The defined background radioactivity in the studied lakes during 1999-2000 was stable, with a level not exceeding $20 \mathrm{mkR} \mathrm{h}^{-1}$, presenting the regional norm and unable to impact lake communities.

\section{Discussion}

The overall diversity is the highest in the lakes Bozshakol, Kamyshovoe, Kojbagor (63 - 112 species), and some other freshwater lakes (Tab. 1) of IV water salinity class.

Bio-indicational analysis of algal diversity shows that the dominant indicator species are alkaliphiles, oligohalobes-indifferents and beta-mesosaprobes conveying the integrity of major ecological variables.

Similarity analysis (Fig. 3) is based on the distribution matrix of 254 revealed species over 34 water bodies and calculated as the percent disagreement by WARD's method. The dendrogram shows that the algal taxonomic diversity is divided into three different clusters, with most of the Kazakhstan lakes separated at the $74 \%$ similarity level. The group designated at the $82 \%$ similarity level (cluster 2) comprises algal assemblages of lakes with great amplitude of salinity fluctuations (II to IV classes), with species numbers $1-28$. The dominant indicators are oligohalobes-indifferent, halophiles and mesohalobes.

The second group discriminated at $82 \%$ similarity level comprises assemblages of moderately mineralized lakes of III-IV salinity classes, with the species numbers $18-37$. The dominant indicators are oligohalobes-indifferent, halophiles and occasionally mesohalobes.

Cluster 3 of low similarity level comprises assemblages of slightly mineralized lakes of IV salinity class: Bozshakol, Tuntugur, Jaman, Teniz, Kamyshovoe, and Kojbagor, with species numbers 57 (Jaman) to 112 (Kojbagor), dominated by oligohalobes-indifferent and halophiles; mesohalobes are lacking in these lakes.

Therefore, the dendrogram clustered all the revealed diversity around three major variables: species richness of algal communities, salinity class, and the dominant salinity indicators. The most similar are the species-rich communities of slightly mineralized lakes, as well as the species-poor communities of highly mineralized lakes (Ács et al. 2003). These regularities indicate that, other conditions remaining the same, salinity is the main depressing factor of algal diversity irrespective of the type and distribution of the water body. In other words, the compositions of algal communities reflect in the first place the salinity level related to climatic aridity.

Because the species diversity in protected wetlands is mostly influenced by natural factors, floristic cores can reflect historical natural impact on algal biodiversity. Comparative floristics help summarize regional algal diversity in major floristic cores (Fig. 4). We used comparative floristic approaches also for revealing the major factors influencing the lacustrine flora enriching process. In the statistical program GRAPHS (NOVAKOVSKY 2004) which presented not only tables of calculation but also constructed visual graphs, we ana- 


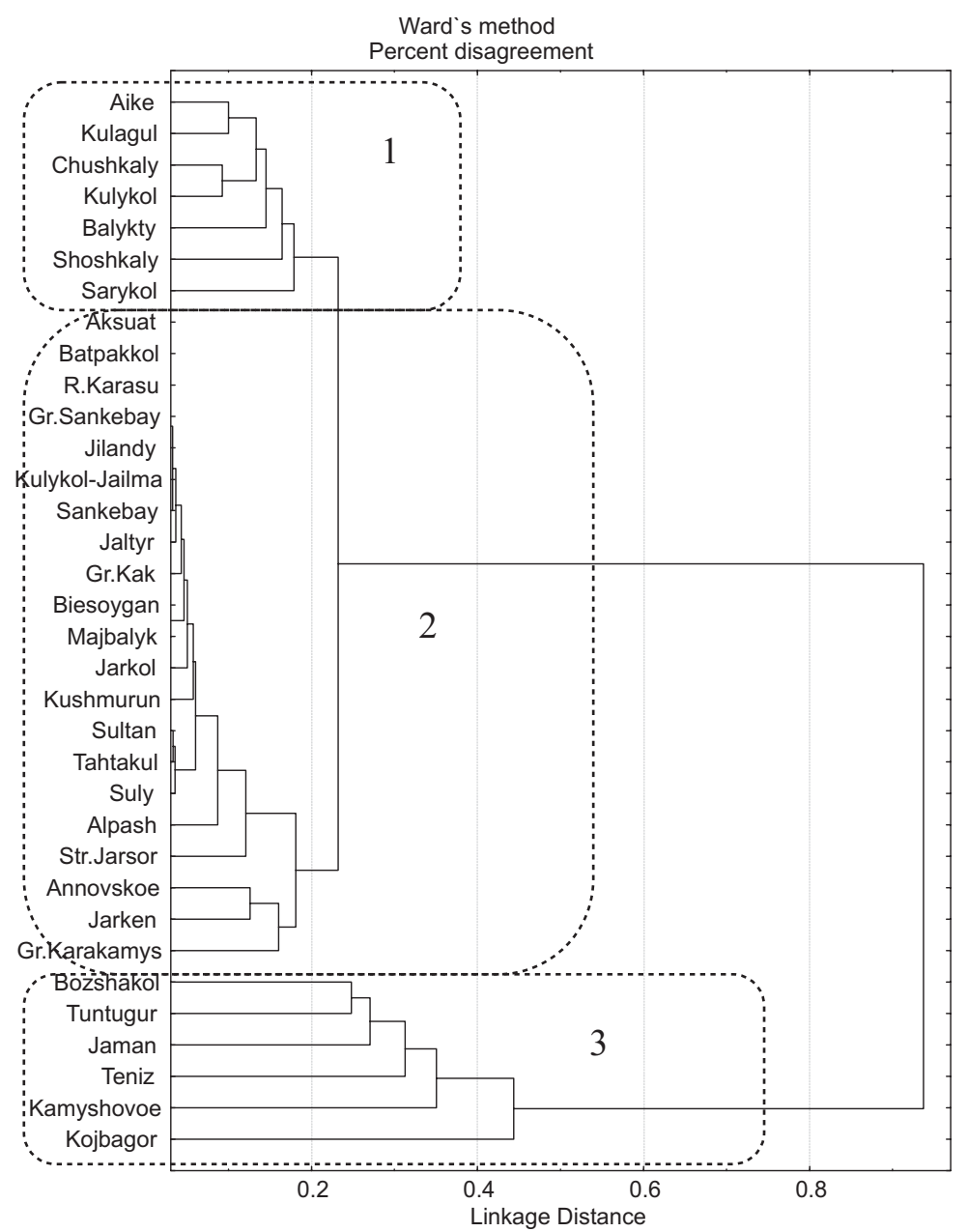

Fig. 3. Tree diagram for algal species diversity in the wetland lakes of Kazakhstan, WARD's method, percent disagreement.

lyzed presence-absence of 254 species in 34 lakes with SERENSEN-CHEKANOVSKY indices calculation. As a result, a dendrite of similarity (Fig. 4) shows five floristic cores, which are marked by dashed lines.

Most lakes with species rich communities and fresh water combined into central core (A). The lakes Bozshakol with 63 species and Kojbagor with 112 species placed in the center of core A. All lakes from core (A) are 3-4 salinity class with high species diversity, low to medium dissolved solids and seasonally fluctuating electrical conductivity, neutral to low acidic range of $\mathrm{pH}$, low to middle nutrient concentration, and III-IV class of water pollution. This means that ecosystems in core (A) lakes are well developed.

Core (B) formed 9 freshwater lakes with middle species diversity, medium dissolved solids and nutrients concentration, clearer than in core (A), but with neutral to low alkalic water. 


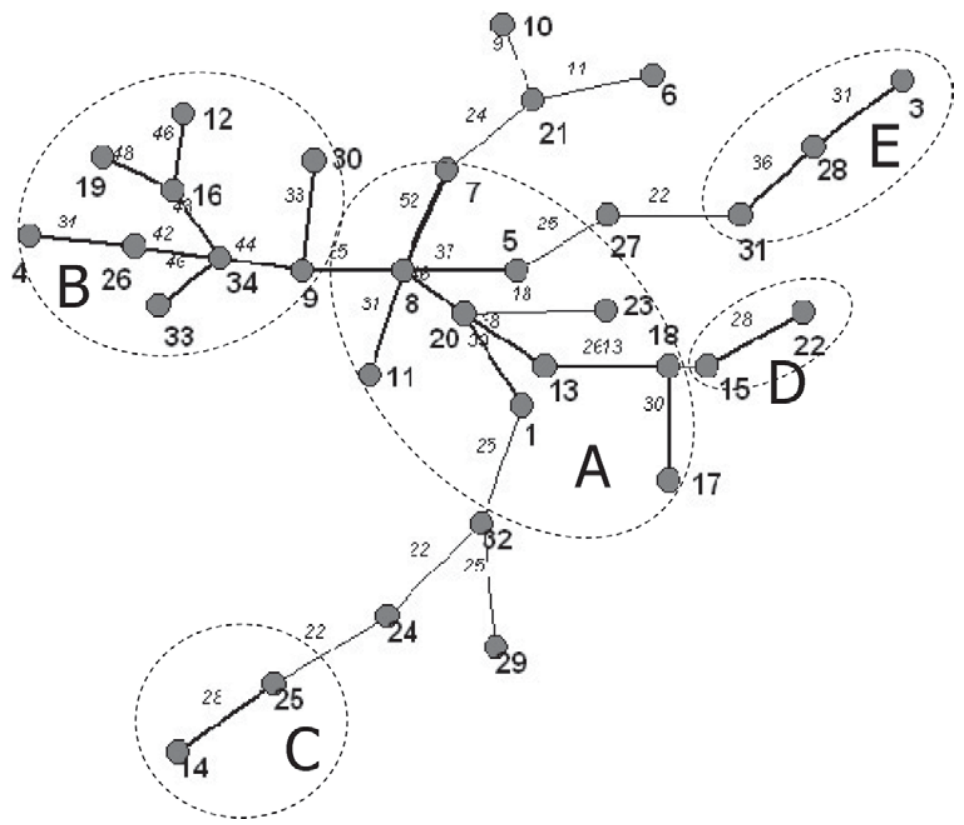

Fig. 4. Dendrite of similarity constructed on the base of SERENSEN-CHECKANOVSKY indices.

Core (C) included two lakes only that connected with core (A) and characterized as freshwater, low alkaline, low organic polluted with low to medium dissolved solids and nutrients concentration.

Core (D) also formed two lakes, which have conditions similar to those lakes of core (C) and also closely related with the diversity of core (A).

The last core $(\mathrm{E})$ included three freshwater lakes, which have similar conditions with lakes from the major core (A).

A few lakes that are not included in the mentioned above cores have intermediate (as in Tahtakol or Kushmurun) or extreme environmental conditions such as in the Great Kak Lake: high salinity and electrical conductivity, low acidic water with low phosphates and medium nitrates concentration and as a result low species diversity.

Therefore, comparative floristic analysis pointed to salinity as the most important factor that has had a historical influence on algal diversity in the studied wetland lakes.

The assessment of the aquatic ecosystems state is based on a correlation of hydrochemical data and their ranges for nitrogen and phosphorus (the trophic elements) with those for saprobity indices (the biota's self-purification capacity). We calculated the index of the water ecosystem state, WESI (BARINOVA 2000, BARINOVA et al. 2006) relating the ranges of self-purification to those of trophic elements. WESI reflects the potentials of the aquatic ecosystem to regenerate after anthropogenic impacts. It also conveys the intensity of anthropogenic impacts if such occurred. In the case of WESI above or equal to 1.0, the ecosystem is assessed as balanced and buffered from anthropogenic impacts. At WESI below 1.0, the biotic components are under toxic influences. A smaller WESI reflects a greater toxicity. Sometimes the latter can be natural rather than anthropogenic. 
Our analysis showed a normal (balanced) state for the ecosystems of the lakes Kulykol, the spring Jailma, the spring Jarsor and Sarykol Lake (Tab. 4) in dry autumn of 1999. Most of the samples reveal a slight natural toxic influence which may be come from sulphides. More significant toxic influence is recognized for the lakes Aike, Sankebay, Jarkol, and Bozshakol. However, the composition of their algal communities gives no evidence of heterotrophy.

Normal state (WESI above or equal 1.0) was found in the spring season of 2000 in the lakes Alpash, Kulykol, Balykty, Kulagul, Great Sankebay, Kojbagor, Tuntugur, Teniz, Sultan, Jarken, Sarykol, Kamyshovoe, Jaman, Shoshkaly, Karakamys, Aksuat, and the stream Jarsor. This group includes most of the lakes. At the same time, a slight toxic suppression of photosynthetic activity was revealed for the lakes Suly, Majbalyk, Jarkol, Chushkaly, Taunsor (Teniz), Bozshakol, Jaltyr, Jilandy, Annovskoe, Tahtakul, Sarybalyk, Karakamys. Therefore, the toxicity might have been temporary, during the dry period, unrelated to any constant anthropogenic impact. According to the functional model of aquatic ecosystems (BARINOVA 2000, BARINOVA et al. 2006), ecosystems of majority of lakes are at the regenerating stage and most influenced by evaporation in summer season.

Algal species and index saprobity S dynamic in communities during the 1999-2000 study period show that the maximal taxonomic diversity (number of species) for algal communities in 1999 was observed in the lakes Kojbagor (64), Bozshakol (30), Kamyshovoe (53) and Jaman (40). The saprobity index S varied from 1.48 to 2.70, which corresponds to 2b-4a ranks of water quality. Although sulfides were periodically revealed in Bozshakol Lake, algal species richness is rather high because this lake is fresh and provides the best environment not only for higher aquatic plant development, but also for algal diversity in plankton and submerged plants. The means of $S$ for these lakes in the spring revealed a high self-purification capacity.

The most species-rich in the year 2000 were the lakes Balykty (26), Tounsor (Teniz) (30), Kojbagor (64), Tuntugur, Bozshakol (30), Kamyshovoe (53), Jaman (40), and other swith more than 20 species per each sample.

On the basis of nitrate concentration, most of the lakes in 1999 were assigned to $4 \mathrm{~b}$ and 5a-b water quality ranges (Tab. 3), which indicates a reduced consumption of this element by the lacustrine biota. For phosphate concentrations, 19 samples fall in the same high ranges. Only in the Kulagul (Kulykol) Lake is there a high concentration of phosphates against the minimal nitrate concentration. In this lake the algal productivity is limited by nitrogen which explains the low consumption of phosphates. During the study period only in the northeastern part of Bozshakol Lake was a high concentration of sulfides $\left(\mathrm{H}_{2} \mathrm{~S}\right)$ found, which can be explained by anaerobic decomposition of dead matter produced by the lake ecosystem during the periods of water bloom or a decay of aquatic macrophytes. This biota toxic variable periodically formed a reduction zone in the bottom, but in the thin layer of water under the surface life is flourishes.

On account of their nitrate and phosphate concentrations, several lakes were assigned to $4 \mathrm{~b}$ and 5a water quality classes in 2000 (Tab. 5), indicating under-consumption of these components by the biota. In lakes Maybalyk, Tounsor (Teniz), Sultan, Bozshakol, Sarykol, Annovskoe, and Sarybalyk, a relatively high concentration of nitrates was associated with a low concentration of phosphates; the nitrates were under-consumed, because the development of algal community was limited by phosphorus. Nitrogen was not a limiting factor 
in these lakes. As a whole, the biotic communities were actively developing, although occasionally restricted by the deficit of phosphorus in freshwater lakes and in a single brackish lake, Sarybalyk.

\section{Conclusion}

The calculated indices of the environmental quality of Kazakhstan's arid region wetlands ranged within the expected natural variations. Toxic influence is recognized in Jaltyr, Jilandy, and Karakamys. A slight natural influence was revealed for most of the lakes. The organic matter enriching the water after aquatic plant death regulates the production of sulfides as toxic substances for algae. Yet the algal communities provide no evidence of heterotrophy. The toxicity might have been temporary, unrelated to a constant anthropogenic impact. According to the functional model of aquatic ecosystems (BARINOVA et al. 2006), lakes Jaltyr, Jilandy, and Karakamys are assigned to the regeneration stage.

In comparison to 1999 and 2000, salinity (mineralization) in the studied lakes slightly decreased (by 0.01-0.02), with the exception of Sarybalyk and Aksuat, where it increased from 1.47 and 0.69 respectively in autumn 1999 to 8.88 and 3.94 respectively in spring 2000 .

Salinity as a consequence of aridization suppressed algal diversity, and thereby decreased the productivity of the first trophic level, undermining the trophic base of wetlands as water fowl habitat.

Lake ecosystems are insignificantly disturbed, only few of them revealing an appreciable toxic effect. The saprobity indices calculated for each of the lakes attest to a high self-purification capacity. By comparing of the ecosystem state indices, WESI, for 1999 and 2000 we are led to the conclusion that self-purification activity increased from dry to wet seasons in Kulagul, Sankebay, Jarkol, Kojbagor, Tuntugur, Bozshakol, Annovskoe, and Tahtakul. A seasonally increase of salinity in lakes Aksuat, Sarybalyk, and Jarsor did not affect their general state.

Therefore, we concluded that salinity in the lakes is the most important factor that has also had a historical influence on the algal diversity of the arid region wetland lakes.

\section{Acknowledgements}

We thank E. A. Bragin, N. N. Berezovikov, S. N. Erokhov, V. S. Vilkov and V. I. Drobovtsev for help in the sampling trips as well as A. G. KARLSEN and A. Solovieva for help in laboratory analysis. This work was partly supported by the Israeli Ministry of Absorption.

\section{References}

Ács, É., Borsodi, A. K., Makk, J., Molnár, P., Mózes, A., Rusznyák, A., Reskóné, M. N., Kiss K. T., 2003: Algological and bacteriological investigations on reed periphyton in lake Velencei, Hungary. Hydrobiologia 506-509, 549-557.

ApHA, Awwa, WeF, 1998. Standard methods for the examination of water and wastewater, Method 4500Cl-B. American Public Health Association and American Water Works Association and Water Environment Federation, Washington, D.C. 
Barber, H. G., CARTER, J. R., 1996: An atlas of British diatoms. Biopress Limited, Dorchester, U.K.

BARINOVA, S. S., 1988: Polymorphism of connective structures in diatom algae. In: Krassilov, V. A. (ed.), Evolutionary research. Vavilov's Themes, 110-122 (in Russian). Academy of Sciences of USSR, Far East Branch, Vladivostok.

BARINOVA, S. S., 1997: Morphology of connective spines in diatom algae of the genus Aulacoseira Thwaites. Paleontological Journal, Moscow 31, 239-245.

BARINOVA, S. S., 2000: Methodical aspects of algal biodiversity analysis. In: BARINOVA, S. S. (ed.), Algae as indicators of environmental assessment, 4-59 (in Russian). Institute of Natural Conservation Press, Moscow.

Barinova, S. S., Medvedeva, L. A., Anissimova, O. V., 2006: Diversity of algal indicators in environmental assessment (In Russian). Pilies Studio, Tel Aviv.

Barinova, S. S., Bragina, T. M., Nevo, E., 2009: Algal species diversity of arid region lakes in Kazakhstan and Israel. Community Ecology 10, 7-16.

Barinova, S. S., Karlsen, A. G., Solovieva, A. A., 2002: Sustainable assessment of some water ecosystems of Kostanai Oblast and west part of North-Kazakhstan Oblast on the hydrochemical and hydrobiological dates. In: BraginA, T. M., Bragin, E. A. (eds.), The most important wetlands of North Kazakhstan (Kostanai Oblast and west part of North-Kazakhstan Oblast), 39-43 (in Russian). Russian University Press, Moscow.

Barinova, S. S., Medvedeva, L. A., Anissimova, O. V., 2000: Ecological and geographical data of algae-indicators. In: BARINOVA, S. S. (ed.), Algae as indicators of environmental assessment (in Russian), 60-150. Institute of Natural Conservation Press, Moscow.

Bragina, T. M., Bragin, E. A. (eds.), 2002: The most important wetlands of North Kazakhstan (Kostanai Oblast and west part of North-Kazakhstan Oblast) (in Russian). Russian University Press, Moscow.

ETTL, H., 1978: Xanthophyceae. 1. Süßwasserflora von Mitteleuropa, 3. G. Fischer, Stuttgart.

Ettl, H., Gartner, G., 1988: Chlorophyta II. Tetrasporales, Chlorococcales, Gloeodendrales. Süßwasserflora von Mitteleuropa, 10. G. Fischer, Stuttgart.

Gollerbach, M. M., Kossinskaya, E. K., Polansky, V. I., 1953: Blue-green algae. Guide to Freshwater Algae of the USSR, 2 (In Russian). Soviet Science Press, Moscow.

Hammer, U. T., 1986: Saline lake ecosystems of the world. Dr W. Junk Publishers, Dordrecht.

Hegewald, E., 2000: New combinations in the genus Desmodesmus (Chlorophyceae, Scenedesmaceae). Algological Studies 96, 1-18.

Hustedt, F., 1938-1939: Systematische und ökologische Untersuchungen über die Diatomeenflora von Java, Bali und Sumatra. Archiv für Hydrobiologie, Supplement 15, 131-177.

Hustedt, F., 1957: Die Diatomeenflora des Flüßsystems der Weser im Gebiet der Hansestadt Bremen. Abhandlungen Naturwissenschaft Verein Bremen 34, 181-440.

KISSELEV, N. A., 1954: Pyrrophyta. Flora plantarum cryptogamarum URSS, 6 (In Russian). Nauka Press, Moscow. 
Kolbe, R., 1927: Zur Ökologie, Morphologie und Systematik der Brackwasserdiatomeen. Die Kieselalgen des Sperenberger Salzgebietes. Pflanzenforschung 7, 1-146.

KomÁreK, J., AnAgnostidis, K., 1989: Modern approach to the classification system of Cyanophytes 4 - Nostocales. Archiv für Hydrobiologie Suppliment 82 (Algological Studies 56), 247-345.

KomÁReK, J. and Anagnostidis, K., 1998: Cyanoprokaryota, 1: Chroococcales. Süßwasserflora von Mitteleuropa 19/1. G. Fischer, Jena.

Korde, N. V., 1956: The methods of biological studies for the bottom deposits of lakes (the field methods of biological analysis) (In Russian). In: ZHADIN, V. I. (Ed.), Freshwater life in USSR, 4, 1, 383-413. Russian Academic Science Press, Moscow and Leningrad.

KöPpen, W., Geiger, R., 1953: Die Klimate der Erde (Map). Neubearbeitung 1953 von R. Geiger u. W. Pohl J. Perthes, Gotha.

Krammer, K., 2000: Diatoms of Europe, 4. A.R.G. Gantner Verlag K.G., Königstein.

KRAMMER, K., 1985: Morphologische und lichtmikroskopische Merkmale in Mikrometer bereich. Ein Fergleich. Mikrokosmos 74, 105-109.

Krammer, K., LANGE-Bertalot, H., 1991a: Bacillariophyceae, 1. Naviculaceae. Süßwasserflora von Mitteleuropa, 2/1. G. Fischer, Jena.

Krammer, K., Lange-Bertalot, H., 1991b: Bacillariophyceae, 2. Bacillariaceae, Epithemiaceae, Surirellaceae. Süßwasserflora von Mitteleuropa, 2/2. G. Fischer, Jena.

Krammer, K., Lange-Bertalot, H., 1991c: Bacillariophyceae, 3. Centrales, Fragilariaceae, Eunotiaceae. Süßwasserflora von Mitteleuropa, 2/3. G. Fischer, Stuttgart.

Krammer, K., Lange-Bertalot, H., 1991d: Bacillariophyceae, 4. Achnanthaceae, Kritische Erganzungen zu Navicula (Lineolatae) und Gomphonema Gesammtliteraturverzeichnis, 1-4. Süßwasserflora von Mitteleuropa, 2/4. G. Fischer, Stuttgart.

Lange-Bertalot, H., Krammer, K., 1987: Bacillariaceae, Epithemiaceae, Surirellaceae. Neue und wenig bekannte Taxa, neue Kombinationen und Synonyme sowie Bemerkungen und Erganzungen zu den Naviculaceae. Bibliotheca Diatomologica 15, 1-289.

MATTOX, K. R., STEWART, R. D., 1984: Classification on the green algae: a concept based on comparative cytology. In: Irvine, D. E. G., JoHn, D. M. (eds.), Systematics of the green algae, 29-72. Systematics Association Special Volume 27, Academic Press, London and Orlando.

MEFFERT, M. E., 1987: Planktic unsheathed filaments (Cyanophyceae) with polar and central gas-vacuoles, I. Their morphology and taxonomy. Archiv für Hydrobiologie Supplement 76, 315-346.

Moshkova, N. A., Gollerbach, M. M., 1986: Green Algae. Chlorophyta: Ulotrichophyceae, 1, Ulotrichales. Flora plantarum cryptogamarum URSS, 10 (In Russian). Nauka Press, Leningrad.

Novakovsky, A. B., 2004: Abilities and base principles of program module »GRAPHS « (In Russian). Scientific reports of Komi Scientific Center, Ural Division of the Russian Academy of Sciences 27, 1-28.

Palamar-Mordvintseva, G. M., 1982: Chlorophyta: Conjugatophyceae. Desmidiales. Flora plantarum cryptogamarum URSS, 11 (In Russian). Nauka Press, Leningrad. 
Pantle, E., Buck, H., 1955: Die biologische Uberwachung der Gewässer und die Darstellung der Ergebnisse. Gas- und Wasserfach 96, 1-604.

Popova, T. G., 1966: Euglenophyta. Flora plantarum cryptogamarum URSS, 8 (In Russian). Nauka Press, Moscow.

Popovsky, J., Pfiester, L. A., 1990: Dinophyceae (Dinoflagellida). Süßwasserflora von Mitteleuropa, 6. Gustav Fisher Verlag, Stuttgart.

Romanenko, V. D., OKsijuk, O. P., Zhukinsky, V. N., Stolberg, F. V., LAVRik, V. I., 1990: Ecological impact assessment of hydrotechnical constructions on water bodies (In Russian). Naukova Dumka, Kiev.

Rumrich, U., Lange-Bertalot, H., Rumrich, M., 2000: Diatoms of the Andes from Venezuela to Patagonia/Tierra del Fuego. Gantner Verlag, Ruggell.

Rusznyák, A., Vladár, P., Molnár, P., Reskóné, N. M., Kiss, G., Márialigeti, K., Borsodi, A. K., 2008: Cultivable bacterial composition and BIOLOG catabolic diversity of biofilm communities developed on Phragmites australis. Aquatic Botany 88, 211-218.

SKLIARENKO, S. L., 2006: Researches on key ornithological territories in Kazakhstan and Central Asia. (In Russian). Biodiversity safety of Kazakhstan Association Press, Almaty.

SLÁDEČEK, V., 1973: System of water quality from the biological point of view. Ergebnisse Limnologie 7, 1-128.

SLÁDEČEK, V., 1986: Diatoms as indicators of organic pollution. Acta Hydrochemica und Hydrobiologica 14, 555-566.

Starmach, K., 1985: Chrysophyceae und Haptophyceae. Süßwasserflora von Mitteleuropa, 1. G. Fischer, Stuttgart.

SUBYANI, A. M., 2005: Hydrochemical identification and salinity problem of ground-water in Wadi Yalamlam basin, Western Saudi Arabia. Journal Arid Environments 60, 53-66.

SwIFT, E., 1967: Cleaning diatom frustules with ultraviolet radiation and peroxide. Phycologia 6, 161-163.

TAKHTAJAN, A., 1978: The floristic regions of the world (In Russian). (In Russian). Nauka Press, Leningrad.

Vinogradova, K. L., Gollerbach, M. M., Zauer, L. M., Sdobnikova, N. V., 1980: Chlorophyta, Rhodophyta, Phaeophyta. Flora plantarum cryptogamarum URSS, 13 (In Russian). Nauka Press, Leningrad.

Watanabe, T., Asai, K., Houki, A., 1986: Numerical estimation to organic pollution of flowing water by using the epilithic diatom assemblage - Diatom Assemblage Index (DAIpo). The Science of the Total Environment 55, 209-218.

Whitton, B. A., RotT, E., Friedrich, G., 1991: Use of algae for monitoring rivers. Institute of Botany. Innsbruck University Press, Innsbruck. 